\title{
Neural Markers of Responsiveness to the Environment in Human Sleep
}

\author{
ㅈThomas Andrillon, ${ }^{1,2}$ Andreas Trier Poulsen, ${ }^{3}$ Lars Kai Hansen, ${ }^{3}$ Damien Léger, ${ }^{4}$ and Sid Kouider ${ }^{1}$ \\ ${ }^{1}$ Brain and Consciousness Group, Département d'Études Cognitives, École Normale Supérieure, PSL Research University, 75005 Paris, France, ${ }^{2}$ École \\ Doctorale Cerveau Cognition Comportement, Université Pierre et Marie Curie, 75005 Paris, France, ${ }^{3}$ Technical University of Denmark, DTU Compute, 2800 \\ Lyngby, Denmark, and ${ }^{4}$ Université Paris Descartes, Sorbonne Paris Cité, APHP, Hôtel Dieu, Centre du Sommeil et de la Vigilance et EA 7330 VIFASOM, \\ 75004 Paris, France
}

Sleep is characterized by a loss of behavioral responsiveness. However, recent research has shown that the sleeping brain is not completely disconnected from its environment. How neural activity constrains the ability to process sensory information while asleep is yet unclear. Here, we instructed human volunteers to classify words with lateralized hand responses while falling asleep. Using an electroencephalographic (EEG) marker of motor preparation, we show how responsiveness is modulated across sleep. These modulations are tracked using classic event-related potential analyses complemented by Lempel-Ziv complexity (LZc), a measure shown to track arousal in sleep and anesthesia. Neural activity related to the semantic content of stimuli was conserved in light non-rapid eye movement (NREM) sleep. However, these processes were suppressed in deep NREM sleep and, importantly, also in REM sleep, despite the recovery of wake-like neural activity in the latter. In NREM sleep, sensory activations were counterbalanced by evoked down states, which, when present, blocked further processing of external information. In addition, responsiveness markers correlated positively with baseline complexity, which could be related to modulation in sleep depth. In REM sleep, however, this relationship was reversed. We therefore propose that, in REM sleep, endogenously generated processes compete with the processing of external input. Sleep can thus be seen as a self-regulated process in which external information can be processed in lighter stages but suppressed in deeper stages. Last, our results suggest drastically different gating mechanisms in NREM and REM sleep.

Key words: complexity; EEG; NREM; REM; sensory processing; sleep

\section{Significance Statement}

Previous research has tempered the notion that sleepers are isolated from their environment. Here, we pushed this idea forward and examined, across all sleep stages, the brain's ability to flexibly process sensory information, up to the decision level. We extracted an EEG marker of motor preparation to determine the completion of the sensory processing chain and explored how it is constrained by baseline and evoked neural activity. In NREM sleep, slow waves elicited by stimuli appeared to block response preparation. We also used a novel analytic approach (Lempel-Ziv complexity) and showed that the ability to process external information correlates with neural complexity. A reversal of the correlation between complexity and motor indices in REM sleep suggests drastically different gating mechanisms across sleep stages.

\section{Introduction}

Sleep can be defined as a state of behavioral unresponsiveness (Peigneux et al., 2001), but its extent and underlying mechanisms need to be further specified. Disconnection from the external

\footnotetext{
Received March 18, 2016; revised May 11, 2016; accepted May 14, 2016

Author contributions: T.A. and S.K. designed research; T.A., D.L., and S.K. performed research; T.A., A.T.P., and L.K.H. analyzed data; T.A., A.T.P., L.K.H., D.L., and S.K. wrote the paper.

This work was supported by Agence Nationale de la Recherche Grants ANR-10-LABX-0087 and ANR-10-IDEX0001-02, European Research Council Project METAWARE to S.K., and Ministère de la Recherche and the Société Française de Recherche et Médecine du Sommeil to T.A. We thank Hernan Anllo, Leonardo Barbosa, Virginie Bayon, Audrey Dalbin, Isabelle Dautriche, Livio de Sanctis, Maxime Elbaz, Louise Goupil, Stéphane Rio, and Chiara Varazzani for their help.

The authors declare no competing financial interests.
}

world may play a crucial role in memory consolidation, allowing the brain to turn inward and protect endogenous mechanisms of neural plasticity from external interferences (Rasch and Born, 2013). Yet, this disconnection potentially comes at the expense of survival, as a sleeping organism becomes highly vulnerable. It remains unclear, however, to which extent such disconnection is implemented and modulated. The influential thalamic gating

Correspondence should be addressed to Dr. Thomas Andrillon, Brain and Consciousness Group, Département d'Études Cognitives, 29 rue d'Ulm, École Normale Supérieure, PSL Research University, 75005 Paris, France. E-mail: thomas.andrillon@gmail.com.

DOI:10.1523/JNEUROSCI.0902-16.2016

Copyright $\odot 2016$ the authors $\quad 0270-6474 / 16 / 366583-14 \$ 15.00 / 0$ 
hypothesis proposed that behavioral unresponsiveness was achieved at an early stage, through the blockade of sensory information at the thalamic level (McCormick and Bal, 1994). However, familiar and salient stimuli trigger an awakening more easily (Oswald et al., 1960; Formby, 1967), suggesting the preservation of basic sensory processes. Even when sleep is preserved, familiar stimuli exhibit different brain responses (Perrin et al., 1999). In recent years, several studies have shown that external information processing may extend far beyond automatic operations: from the detection of semantic incongruity (Bastuji and GarcíaLarrea, 1999; Ibáñez et al., 2006) or the violation of simple rules (Ruby et al., 2008; Strauss et al., 2015) to the formation of new associations (Arzi et al., 2012; de Lavilléon et al., 2015). The cognitive processes involved during sleep therefore appear more elaborated than previously thought.

However, although these studies have demonstrated the preservation of cognitive abilities during sleep, the neural mechanisms allowing sleepers to process or isolate from external stimulations remain unsettled. The role of evoked slow oscillations, such as K-complexes, a hallmark of NREM sleep, is particularly unclear (Halász, 2005). K-complexes have sometimes been related to arousal systems (Siclari et al., 2014) providing windows of wakefulness to sleepers (Destexhe et al., 2007). Conversely, they have also been described as local down states (Cash et al., 2009) entailing large-scale neuronal silencing (Vyazovskiy and Harris, 2013) and protecting sleep against external stimulations (Wauquier et al., 1995; Bastien et al., 2000). This tendency neurons have to alternate between periods of activations (up states) and silencing (down states) (Sanchez-Vives and McCormick, 2000 ) is termed "neuronal bistability" and has been proposed as a mechanism limiting sensory processing at the cortical level (Tononi and Massimini, 2008; Pigorini et al., 2015). Sleep spindles are also thought to enable sleeper's isolation by blocking incoming input at the thalamic level (McCormick and Bal, 1994). However, evidence supporting this hypothesis is scarce (Schabus et al., 2012), whereas cellular recordings showed remarkably preserved responses to sounds during spindles (Sela et al., 2016).

REM sleep, on the other hand, has been far less studied. Paradoxically, even if brain activity in REM sleep resembles wakefulness and consciousness is regained (Hobson and Pace-Schott, 2002), sleepers remain largely unresponsive (Ermis et al., 2010). It has been proposed that dreams themselves would compete and block the processing of external inputs (Nir and Tononi, 2010), but direct evidence is still missing.

Here, to explore responsiveness during human sleep, we relied on a paradigm that aimed at inducing task-dependent responses in the sleeping brain during naps (Kouider et al., 2014). In practice, participants fell asleep while categorizing spoken words with lateralized responses. Using EEG recordings, we explored whether stimuli elicited brain activations corresponding to the motor preparation of the correct response (i.e., lateralized readiness potential [LRP]) (Masaki et al., 2004; Smulders et al., 2012). The presence of such LRP implies that stimuli were not only encoded at the sensory level but also processed at a high level of semantic representation and propagated up to the preparation of a motor response (see Kouider et al., 2014). The presence of an LRP highlights the maintenance of complex distributed processes during sleep. Although our previous study was limited to daytime sleep (nap study), here we investigated all sleep stages in a full-night protocol. Furthermore, the presence and magnitude of the LRP was further evaluated in light of brain activity before and after the stimulus so as to provide novel insights on how the brain manages to alternatively track its environment or rather preserve sleep.

\section{Materials and Methods}

\section{Participants}

Twenty-three right-handed French native speakers (16 females, age 21-31 years) with no history of neurological or sleep disorders participated to this study. Participants underwent an interview with a sleep specialist and filled in questionnaires determining their sleep habits and their propensity to fall asleep in noisy environments. Participants did not complain of any sleep disorder. They were monitored for 7-10 d before the recording session through actigraphy and sleep diaries to ensure regular sleep/wake rhythms. Among these 23 participants, 5 participants ( 4 females) were discarded from our analyses either for technical issues affecting the recordings $(N=1)$ or because the experiment was aborted $(N=4$, participants experiencing difficulties to fall asleep with auditory stimulations). The protocol had been approved by the local ethics committee (Comité de Protection des Personnes, Ile-de-France I, Paris, France).

\section{Experimental procedure}

Task. We adapted a procedure previously used during daytime naps (Kouider et al., 2014) to a full-night protocol to explore all sleep stages. On the night of the recordings, participants were equipped for polysomnographic recordings. They went to bed, and spoken words in French were then played in isolation one after the other. These words referred either to an animal or to an object. Participants were instructed to perform a semantic-decision task by indicating the category of each word through right- and left-hand responses. They were asked to categorize words as long as they were awake and to resume responding in case of an awakening. Subjects were reminded to do so whenever they awoke during the night without resuming to respond. However, participants were explicitly authorized to fall asleep while performing the task. Crucially, three different lists of words were played to participants according to their vigilance state, as assessed through an online assessment of sleep stages (see below). A list was played whenever participants were in NREM sleep, another list was played during REM sleep, and a wake list was played otherwise (i.e., mainly during wakefulness). Thus, unpracticed words (i.e., novel words not previously categorized during wakefulness) were played in sleep to ensure that participants had to access the meaning of the word to prepare for the appropriate response, without relying on stimulus-response associations learned while awake.

Stimuli. Stimuli were French spoken words uttered by a female voice. Lists of 72 words were created each containing 36 words referring to animals and 36 words referring to objects. Animal and object words were matched in frequency and number of syllables using the Lexique database (New et al., 2004). The attribution of the different lists to a given vigilance state was counterbalanced across participants. Participants received on average $21.7 \pm 1.0$ (mean \pm SEM across participants), $25.2 \pm 0.7$, and $6.9 \pm 0.3$ times the words from the wake, NREM, and REM lists, respectively.

Apparatus. Response-handles were attached to participants' hands. The mapping between semantic categories and response-hands was counterbalanced across participants. Stimuli were played through loud speakers at $\sim 55 \mathrm{~dB}$ (depending on participants' preferences) using the Psychtoolbox extension (Brainard, 1997) for MATLAB (The MathWorks). Stimuli were played every 6-9 s (random uniform jitter).

\section{Data analysis}

Electrophysiological recordings. EEG ( $N=19$ derivations, 10-20 montage), electro-occulographic (EOG, $N=2$ derivations, positioned above the right canthus and under the left canthus), electromyograhpic (EMG, 1 derivation placed on the chin measuring muscle tone and 2 derivations on the right and left abductor pollicis brevis [thumb flexor muscle] recording muscle activity accompanying participants' responses) and electrocardiographic (ECG, $N=1$ derivation) data were continuously recorded. Video monitoring was also available. EEG, EOG, ECG, and EMG data were recorded with $\mathrm{AgCl}$ electrodes attached to participants' skin and hair with an adhesive paste (EC2, Natus Neurology). Signals 


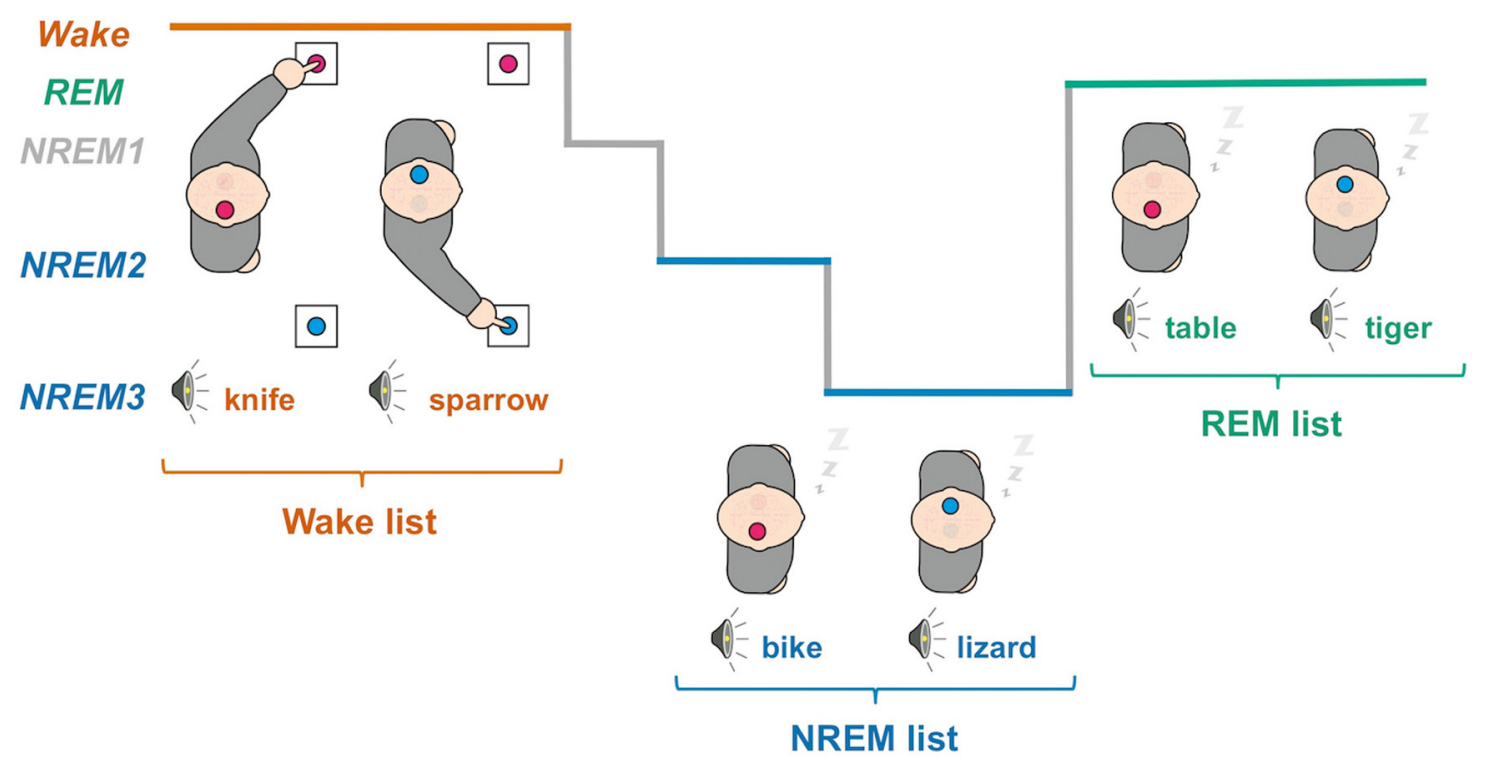

Figure 1. Experimental procedure. Illustration of the protocol. Different lists of animal and object words were played to participants. Participants were instructed to classify these words through left- and right-hand responses according to their semantic category (here right-hand responses for animals). Lateralized hand-response preparation involves the contralateral motor cortices, a task-dependent lateralization of brain activity that can be tracked with the EEG (Fig. 2). Different lists of words were presented to participants. A list was restricted to NREM sleep (NREM2 and NREM3, blue) and another one to REM sleep (green) while the wake list (red) was played otherwise. Changing list between wake and sleep prevents sleepers from using stimulus-response associations learned in wake to classify words in sleep. Words being novel, participants must have had access to the meaning of each word to prepare for the correct response.

were amplified through a B1IP or B2IP MEDATEC amplifier (Medical Data Technology) and recorded at a $200 \mathrm{~Hz}$ sampling rate. Impedances of scalp electrodes were generally $<5 \mathrm{k} \Omega$. EEG and EOG electrodes were referenced online to the opposite mastoids. An additional channel was used to synchronize EEG data with stimulus presentation.

Online sleep assessment. The presentation of stimuli was continuous, but the words presented to participants were selected from lists that differed depending on participant's vigilance state (Fig. 1). Vigilance and sleep states were scored online following standard guidelines (Iber et al., 2007). In practice, the experimenter waited for the appearance of NREM hallmarks (first spontaneous K-complex or sleep spindle in the absence of arousal) before switching to the NREM list. Similarly, the REM list was presented to participants when they entered the REM stage (absence of slow oscillations or sleep spindles, absence of alpha oscillations, presence of saw-tooth waves, rapid eye movements, increase in theta oscillations, reduced or absent muscle tone). The experimenter typically waited for a few minutes after the transition to the new sleep stage before switching the list, so as to ensure sleep stage's stability. The wake list was presented to participants whenever they showed signs of arousal (body movements, increase in low-amplitude desynchronized rhythms, EMG activation). This online scoring was confirmed and refined offline (see below).

Offline sleep scoring. Wakefulness and sleep stages were scored by 2 scorers blind to experimental conditions and following established guidelines (Iber et al., 2007). Only Fz, C3, C4, and Pz EEG derivations (within the 10-20 montage) were used along ECG and EMG derivations. EEG channels were first rereferenced to the average mastoids. The EEG and EOG signals were high-pass filtered $>0.1 \mathrm{~Hz}$ and then low-pass filtered $<30 \mathrm{~Hz}$ (two-pass Butterworth filters at the fifth order). Using the same filter types, EMG data were bandpass filtered between 60 and 80 Hz. A notch-filter (second order Infinite Impulse Response filter) $\sim 50$ $\mathrm{Hz}$ was used on all channels to reduce line-noise. Vigilance states were continuously scored on 20-s-long windows as wakefulness, NREM sleep Stages 1, 2, and 3 (NREM1, NREM2, and NREM3 stages, respectively), and REM sleep. Importantly, epochs showing signs of arousal (increase in alpha oscillations or oscillations $>16 \mathrm{~Hz}$ lasting $>3 \mathrm{~s}$ ) or microarousal $(<3 \mathrm{~s})$ in association with trial onsets were marked. The corresponding trials were not included in further analyses to avoid potential confounds. Table 1 summarizes sleep scoring across participants.

Slow wave detection. Slow oscillations (sleep slow waves and $\mathrm{K}$-complexes) were detected in NREM sleep using an algorithm that has been presented in details previously (Riedner et al., 2007; Nir et al., 2011). Briefly, slow oscillations were detected for each EEG channel by bandpass filtering the EEG signal between 0.2 and $3 \mathrm{~Hz}$ (two-pass Butterworth filter at the third order). The first-order derivative was used to detect local extrema and identify single waves. Only slow oscillations with a peak-to-peak amplitude exceeding $75 \mu \mathrm{V}$ and a duration of $>0.5 \mathrm{~s}$ and $<2 \mathrm{~s}$ were considered as slow waves. These slow oscillations were used to define light and deep NREM trials (see below).

EEG preprocessing. EEG data were analyzed using a combination of SPM (Functional Imaging Laboratory, University College London, London), FieldTrip (Oostenveld et al., 2011), and EEGlab (Delorme and Makeig, 2004) toolboxes running on MATLAB (The MathWorks). We examined the brain activity in response to sounds by computing eventrelated potentials (ERPs) and LRPs. To do so, the continuous EEG signal referenced to the average mastoids was high-pass filtered $>0.1 \mathrm{~Hz}$ (twopass Butterworth filter at the fifth order) and then segmented on large temporal windows around stimulus onsets $([-14,14]$ s). The EEG signal was then low-pass filtered $<40 \mathrm{~Hz}$ and notch-filtered $\sim 50 \mathrm{~Hz}$ (same filter types as for the sleep scoring) to reduce line noise. Epochs were resized to focus on the activity around stimulus onsets $([-2,7] \mathrm{s})$. Trials with absolute amplitude over central electrodes $(\mathrm{Cz}, \mathrm{C} 3, \mathrm{C} 4)$ exceeding a given threshold were discarded $(150 \mu \mathrm{V}$ in wake and REM sleep, and 200 $\mu \mathrm{V}$ in NREM sleep because NREM sleep contains high-amplitude physiological slow waves).

Conditions of interest. In NREM sleep, brain activity varies immensely: from NREM1 in which sleep hallmarks (slow oscillations and sleep spindles) are not yet visible to NREM3 where they predominate (Iber et al., 2007). First, to ensure that observed sensory processes are not due to (even brief) awakenings, NREM1 stage was discarded as well as trials in which any sign of arousal could be observed in the EEG signal. Second, NREM2 and NREM3 trials were separated according to the presence of slow waves associated with stimuli onset. We thus divided trials in four different conditions of interest: (1) wake: correct trials during which participants were scored as awake and the wake list was played; (2) light NREM sleep: trials scored as NREM2 or NREM3, during which participants were unresponsive and in which the NREM list was played but no slow-wave were detected (on a $[-2,3]$ s window time-locked to stimulus onset); (3) deep NREM sleep: trials scored as NREM2 or NREM3, during which participants were unresponsive and in which the NREM list was played and slow waves were detected (on a $[-2,3]$ s window time-locked 
Table 1. Sleep scoring and conditions of interest ${ }^{a}$

\begin{tabular}{|c|c|c|c|c|c|c|}
\hline & Wake & NREM1 & NREM2 & NREM3 & REM & Total \\
\hline Duration (min) & $88.5( \pm 9.6)$ & $45.3( \pm 5.6)$ & $186.3( \pm 13.4)$ & $79.7( \pm 7.3)$ & $76.9( \pm 6.5)$ & $499.6( \pm 6.6)$ \\
\hline$\%$ of trials & $17.8( \pm 1.6)$ & $9.7( \pm 1.0)$ & $39.6( \pm 2.0)$ & $16.4( \pm 1.4)$ & $16.5( \pm 0.8)$ & 100 \\
\hline Marked arousals & 0 & $12.3( \pm 1.6)$ & $13.4( \pm 1.8)$ & $2.6( \pm 0.5)$ & $7.0( \pm 1.5)$ & $35.8( \pm 3.8)$ \\
\hline
\end{tabular}

${ }^{a}$ Data are mean (SEM); $N=18$. Participants' vigilance was scored according to established guidelines as wakefulness, NREM1, NREM2, NREM3, and REM stages on 20-s-long windows (see Materials and Methods). Duration indicates the average time participants spend in each stage. Each trial was attributed a sleep stage defined as the most conservative scoring obtained on a $[-2,8]$ s window around stimulus onset (\% of trials). Marked arousals indicates the mean number of trials that were rescored as wakefulness due to an arousal.

to stimulus onset); (4) REM sleep: trials scored as REM sleep, during which participants were unresponsive and in which the REM list was played. Thus, light NREM, deep NREM, and REM trials comprised only words that had not been practiced before, when participants were awake and responsive (no wake list word). For each condition, only participants with at least 70 trials were included. On average, there were $174 \pm 20$ trials in the wake condition (mean \pm SEM across $N=17$ participants), $232 \pm 28$ in light NREM sleep $(N=15), 400 \pm 25$ in deep NREM sleep $(N=18)$ and $186 \pm 9$ in REM sleep $(N=18)$. Light NREM trials occurred almost exclusively in NREM2 $(93 \pm 1.2 \%, N=15$ participants), whereas deep NREM trials correspond to trials occurring either in NREM 2 or NREM 3 stages (56\% and $44 \%$, respectively, $\pm 3.5 \%$ in 18 participants). Thus, light NREM sleep trials corresponded to a state in which slow oscillations could be observed (NREM2) but not in the vicinity of stimulus onset, whereas deep NREM sleep trials corresponded to a state in which slow oscillations were observed in association with stimuli regardless of the presence of continuous trains of slow waves (e.g., in NREM3). Nonetheless, we obtained qualitatively similar results (see Results) when defining light and deep NREM sleep as NREM2 and NREM3 stages, respectively. We also examined trials corresponding to the definition of light, deep NREM, and REM sleep, but during which the wake list was presented to sleepers instead of the NREM or REM lists. Indeed, participants were switched to the wake list whenever the experimenter was unsure of the state of the sleeper (later verified off-line), at transitions between states or when the experimenter had to attend to another participant (two participants participated in the experiment on each recording night). Although these trials containing practiced words were not randomly intermixed with the unpracticed words, they were scored offline as either REM or NREM sleep by two scorers blind to the list used and revealed ERPs and power spectra nearly identical to the ones obtained in trials in which the sleep lists were played (see Fig. 3). Thus, these trials allowed us to investigate how practiced (i.e., overtly categorized) words were processed during sleep, although precautions must be taken when interpreting such results (see Discussion). Participants with at least 30 trials per conditions were included for this analysis.

ERPs. ERPs were computed by averaging the EEG signal across trials for a given experimental condition after baseline correction $([-0.2,0] \mathrm{s})$.

LRPs. LRPs allow the monitoring of action selection and preparation (Smulders et al., 2012). LRPs are usually computed with EEG data timelocked to motor responses but can also be computed time-locked to stimuli (Leuthold, 2003; Töllner et al., 2012). In our case, due to the absence of responses during sleep, we computed LRPs on stimuluslocked data. LRPs were computed using ERPs recorded from the right (C4) and left (C3) electrodes placed over motor cortices as follows:

$$
L R P=\frac{\left(C 3_{\text {right-hand }}-C 3_{\text {left-hand }}\right)+\left(C 4_{\text {left-hand }}-C 4_{\text {right-hand }}\right)}{2}
$$

ERPs over C3 and C4 electrodes were computed similarly as described above, except for the baseline correction $([-2,0] \mathrm{s})$. Using this formula, LRPs are characterized by a negative deflection starting before participants' response. In subsequent analyses, we extracted the LRP magnitude over the temporal windows in which significant negative clusters were observed (see Figs. 2, 3). To examine how this LRP magnitude was dynamically related to other markers of responsiveness, we computed LRPs on windows of 60 consecutive left- and right-response trials slid every trial (see Figs. 4, 6, 7). The LRP-negative potential was extracted as a positive value (LRP magnitude) by multiplying the LRP formula by -1 . A similar operation was performed on the N500 and N550 potentials amplitude (see Figs. 6, 7) to extract their magnitude. Other variables of the EEG signal were estimated on the same windows such as the LempelZiv complexity (LZc; see Fig. 4) or ERP components (see Figs. 6, 7). These variables were $z$-score normalized across trials for each participant and then aggregated across participants to examine their correlation.

Time-frequency analyses. To better understand how sleep rhythms impact sensory processing, we computed the time-frequency decomposition of the EEG signal in response to stimuli. To do so, we applied FFT on 1.28 -s-long windows (padding ratio of 2 ) on the preprocessed EEG signal (see above). The resulting power for each frequency and time was expressed as the log-ratio of the power at the corresponding frequency and time over the baseline activity $([-1.5,0] \mathrm{s})$ at the same frequency. LRP magnitude and the activity within the slow waves $([1,6] \mathrm{Hz})$ and spindle $([11,16] \mathrm{Hz})$ bands were extracted on windows of 60 consecutive righthand and left-hand responses.

$L Z c$. The $L Z c$ measures the complexity of a given signal by estimating its compressibility (Ziv and Lempel, 1977). A temporally unpredictable signal will have a low compressibility and therefore a high $\mathrm{LZc}$ value. Such an unpredictable signal will be considered as a highly complex signal. Previous studies have shown that LZc accurately tracks the level of consciousness in patients, healthy subjects under anesthesia and during sleep (Casali et al., 2013; Abásolo et al., 2015; Schartner et al., 2015). For example, when the EEG signal is populated by high-amplitude synchronous slow waves as in deep NREM sleep, the signal becomes more predictable and the associated LZc value decreases compared with wakefulness or REM sleep (see Fig. 4a). To calculate the LZc on the continuous EEG, we implemented the approach developed by Schartner et al. (2015). Thus, the complexity was computed at the sensor level. To filter the raw EEG, the MATLAB FDAtool was used to create equiripple Finite Impulse Response FIR filters with linear phase to avoid phase distortion in the EEG. The EEG was first low-pass filtered $<85 \mathrm{~Hz}$ (111th order) and then notch-filtered $\sim 50 \mathrm{~Hz}$ to reduce line noise (296th order). A surface Laplacian was then applied to the EEG data (BCILAB plug-in for the EEGLAB toolbox) (Delorme and Makeig, 2004; Kothe and Makeig, 2013), with a neighbor count of four, to reduce the influence of volume conduction. The data were then epoched around stimuli onset $([-2,6]$ s), and the LZc was extracted for each trial by integrating the EEG data over all the sensors on 500-ms-long windows slid every $50 \mathrm{~ms}$. Details of the LZc algorithm can be found previously (Schartner et al., 2015). Briefly, for each window, the mean of the EEG signal was subtracted for each sensor and linear trends over the entire epochs were removed. A Hilbert transform was then applied to the signal to extract its envelope. Each channel was then binarized: values above the mean value of the envelope for the corresponding epoch and sensors were coded as 1 , and 0 otherwise. The resulting binary matrix was then reshaped sensor-wise into a vector containing the time-points for all channels. The complexity was computed on such vectors and normalized by the complexity calculated on a randomly shuffled version of the same vector. After normalization, LZc takes values between 1 (minimally compressible, i.e., as predictable as the shuffle data) and 0 (fully compressible, i.e., predictable signal). The normalized complexity was thus computed for each trial and averaged across vigilance states (as scored offline) over the prestimulus baseline $([-1.5,0] \mathrm{s})$ to compare the overall level of complexity between these different states (see Fig. 4a). We also examined how the complexity was affected by stimulation by averaging the LZc on a $[-1.5,6] \mathrm{s}$ windows for each vigilance state separately in an approach similar to when calculating ERPs (see Fig. $4 b$ ). To better compare the dynamics, LZc was normalized (ratio) by the level of complexity in the prestimulus window 


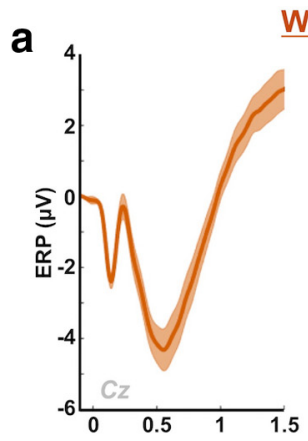

Wake

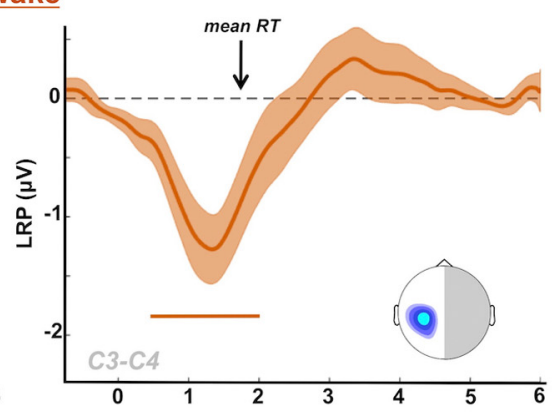

light NREM
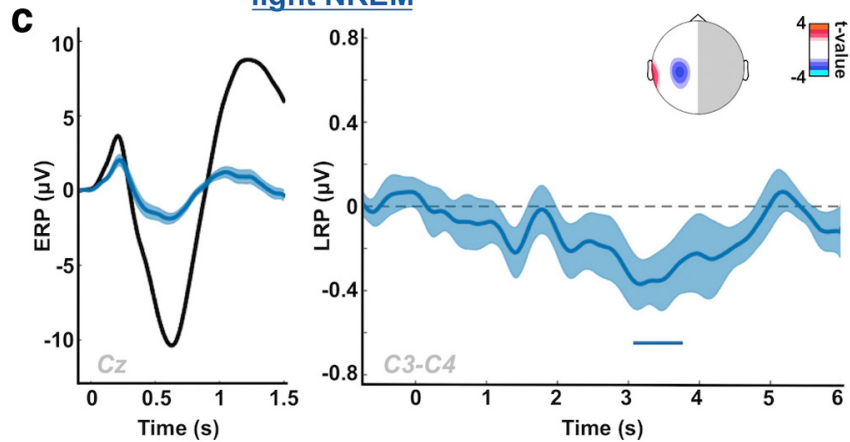

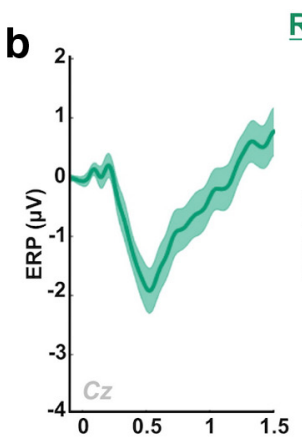

REM

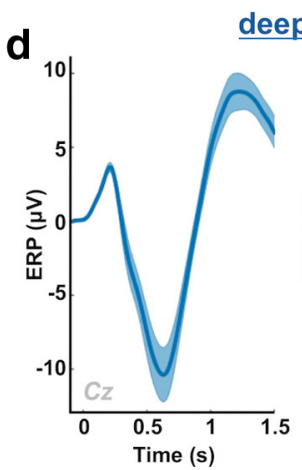

deep NREM

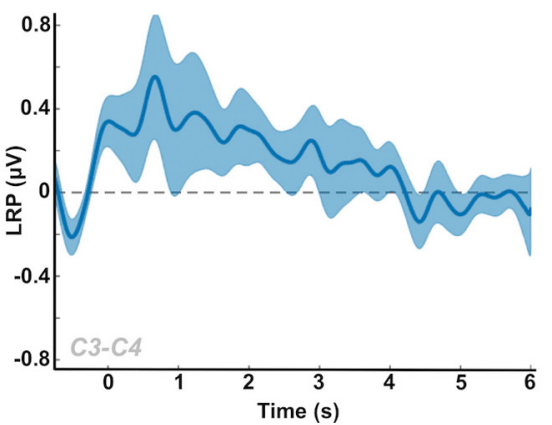

Figure 2. LRPs across sleep stages. The LRP allows monitoring the lateralization of brain activity associated with motor selection and preparation. We used it here as an index of participants' ability to process sensory information up to the semantic level and to use this information in a flexible task-dependent fashion (see Materials and Methods) (Kouider et al., 2014). Subpanels, Left, The stimulus-locked ERP computed on (z: $\boldsymbol{a}$, in wakefulness; $\boldsymbol{b}$, in REM sleep; $\boldsymbol{c}$, in light NREM sleep (blue, black curves show deep NREM sleep for comparison); $\boldsymbol{d}$, in deep NREM sleep. Right, The corresponding stimulus-locked LRP computed on C3/C4 electrodes are plotted. Shaded areas represent the SEM computed across participants. Colored horizontal bars represent significant clusters for LRP ( $p_{\text {cluster }}<0.05$ ). Insets, Scalp topographies of LRP averaged over the red and blue clusters. Curves were smoothed using a Gaussian kernel (width: $50 \mathrm{~ms}$ for ERPs, $200 \mathrm{~ms}$ for LRPs) for display only (statistics were performed before smoothing). An LRP peaking over motor cortices is visible in light NREM but is absent in deeper sleep stages (deep NREM and REM sleep). RT, Response time.

$([-1.5,0] \mathrm{s})$. To relate the level of complexity with the index of motor preparation (LRP magnitude), the baseline LZc was extracted in wakefulness, NREM and REM sleep on windows of 60 consecutive left- and right hand-response trials slid every trial along the entire recordings. The values obtained were then $z$-scored across trials for each participant before examining their correlation (see Fig. $4 c$ ).

Sleep cycle identification. To examine how markers of responsiveness are modulated across NREM sleep, we identified the sleep cycles based on each participant's hypnograms. A total of 100 cycles were individualized in 18 participants ( $5.6 \pm 0.2$ cycle per participant). Values of interest (LRP magnitude, LZc and $\delta(<4 \mathrm{~Hz})$ power) were computed on 60 righthand and left-hand consecutive NREM trials (NREM2 and NREM3) slid every trial within each cycle. Delta power was extracted by applying a FFT of the signal time-locked to stimuli onsets $([-2,6]$ s) and normalizing the $\delta$ range $(<4 \mathrm{~Hz})$ with higher frequencies $([20,40] \mathrm{Hz}$, log ratio). Later on, each sleep cycle was normalized in duration to average variables of interest across sleep cycles. To do so, each cycle was divided in 30 equal bins and the mean value of the variables of interests was computed for each bin. Eighty-three cycles were eventually included in this analysis. The other cycles did not have enough NREM2 and NREM3 trials so as to be similarly normalized in duration.

Statistics. To correct for multiple comparisons when examining statistical differences between two time series (see Figs. 2-5), we used a clusterpermutation approach (Maris and Oostenveld, 2007). Each cluster was constituted by the samples that consecutively passed a specific threshold (here, $p<0.1$ for LRPs and $p<0.05$ otherwise). As demonstrated previously (Maris and Oostenveld, 2007), this method controls for Type 1 errors independently of this threshold. For each cluster, we computed the sum of the $t$ values of all the samples within the cluster. Then, we compared the cluster statistics of each cluster with the maximum cluster statistics of 1000 random permutations and obtained a nonparametric $p$ value (Monte-Carlo $p$ value: $p_{\text {cluster }}$ ). Significant clusters are displayed as horizontal bars on plots, and $p_{\text {cluster }}$ are reported in the main text and figures' legends. For scalp topographies of correlation analyses (Figs. 6,
7), the False Detection Rate (FDR) method was used to correct individual $p$ values (Benjamini and Yekutieli, 2011).

\section{Results}

Sleepers can classify words during sleep

A classical LRP was observed when participants were awake and responsive (Fig. $2 a$; significant cluster: $[0.490,2.025] \mathrm{s}, p_{\text {cluster }}=$ $0.002, N=17$ participants), characterized by a large negative deflection starting before the average response time (black arrow) over motor electrodes (Fig. 2a, inset). When focusing on light NREM sleep, we observed a similar significant negative deflection for the LRP (Fig. $2 c$; significant cluster: $[3.035,3.775] \mathrm{s}, p_{\text {cluster }}=$ $0.01, N=15$ participants), although the corresponding words had not been previously categorized during wakefulness. We obtained a similar LRP even when discarding all words that had been previously presented during an even brief $(<3 \mathrm{~s})$ arousal (cluster: $[2.830,3.310] \mathrm{s}, p_{\text {cluster }}=0.043, N=15$ participants), thus when focusing on words that had been presented exclusively during sleep throughout the entire recording session. As in wakefulness, this negative deflection was maximal over motor cortices (central electrodes; see Fig. $2 c$, inset) but was delayed compared with wake trials. This LRP is strikingly similar to the one observed in previous nap studies (Kouider et al., 2014).

However, no significant deviation in the LRP was observed in deep NREM sleep or REM sleep (Fig. $2 b, d$ ), suggesting, at first glance, that processing of sensory information up to the decision level is abolished in deeper stages of sleep. Interestingly, light and deep NREM sleep also differed regarding the evoked responses to stimuli (ERPs). Light NREM sleep was characterized by a reduction of the large negativity evoked by stimuli in NREM sleep (N550) (Picton, 2010). This N550 is often attributed to evoked 

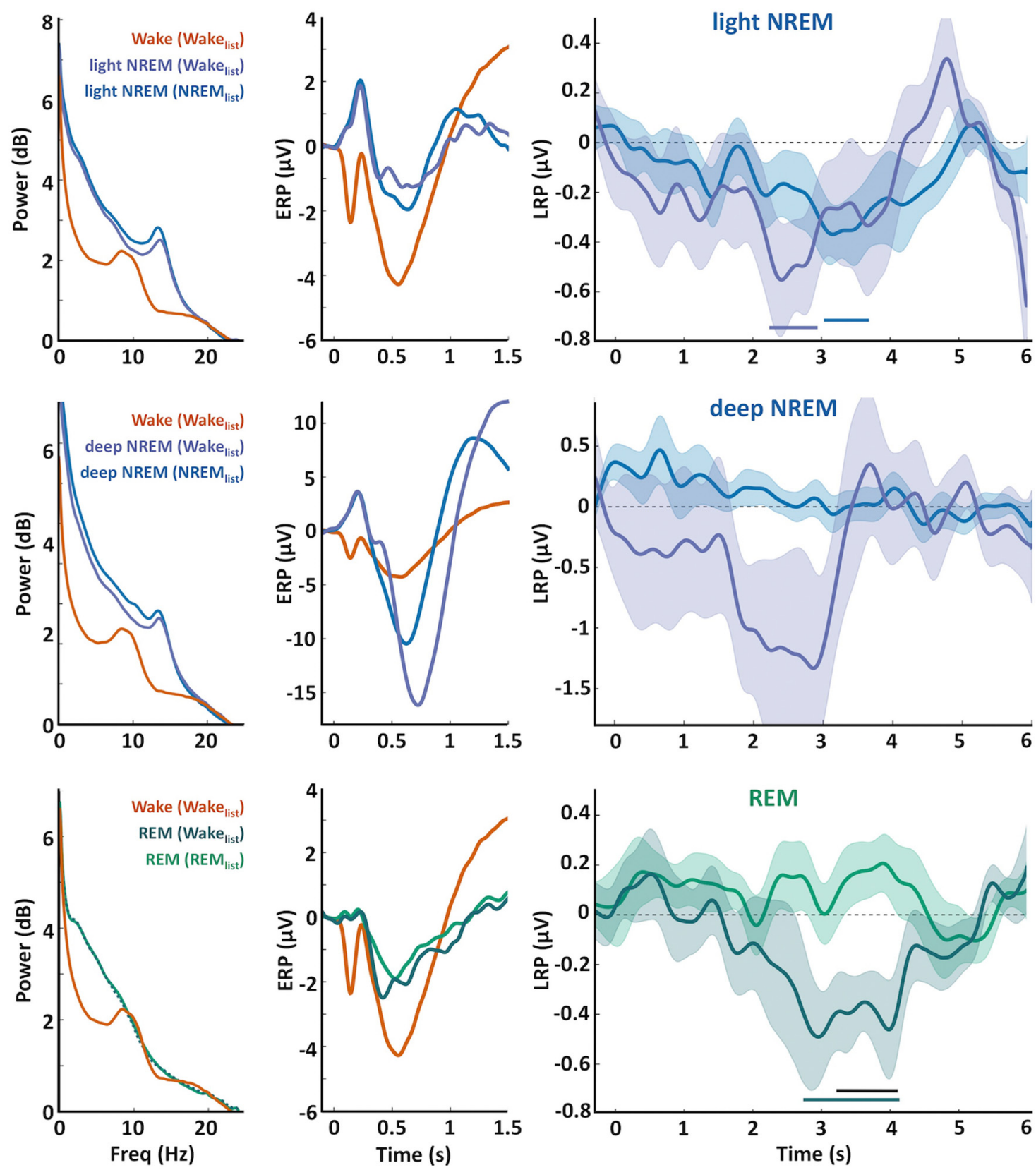

Figure 3. LRPs in light NREM, deep NREM, and REM sleep for words categorized during wakefulness. Top, Power spectra (left), stimulus-locked ERPs (middle), and stimulus-locked LRPs (right) computed in light NREM sleep for trials in which either the NREM list (blue) or the wake (purple) list was played. The wake ERP and power spectrum (red curves) are displayed for comparison. Horizontal bars represent the significant clusters for LRPs $\left(p_{\text {cluster }}<0.05\right)$. Note the presence of a similar (and slightly earlier) LRP when words categorized during wakefulness were played. Middle, Same plots for deep NREM sleep trials. No significant LRP cluster could be observed for either the NREM or the wake lists. Bottom, Power spectra (left), ERPs (middle), and LRPs (right) computed in REM sleep for trials in which either the REM list (light green) or the wake (dark green) list was played. Interestingly, for words previously categorized in wakefulness (practiced), a clear LRP was observed $\left(p_{\text {cluster }}<0.05\right.$ ) but not for unpracticed words (black bar represents cluster for the comparison between the LRPs for the wake and REM lists, $p_{\text {cluster }}<0.05$ ). Yet, the power spectrum and ERPs for both practiced and unpracticed words are highly similar and different from wake trials (red curves). Shaded areas represent the SEM computed across participants.

slow waves (such as K-complexes) (Bastien et al., 2000), which entails large-scale neuronal silencing (Vyazovskiy and Harris, 2013) and could explain the absence of an LRP when the N550 is larger. Importantly, defining light and deep NREM sleep more classically as NREM2 and NREM3 stages, respectively, led to similar results (i.e., presence of an LRP in light NREM $([4.1,4.6] \mathrm{s}$, $\left.p_{\text {cluster }}=0.038\right)$ and absence in deep NREM).

\section{In REM sleep, only words previously heard in wake} are classified

Does the absence of an LRP in REM sleep mean that stimuli were not processed at all, or is this absence due to the complexity of the task at hand? To investigate this issue, we took advantage of the fact that words from the wake list were sometimes presented in REM sleep (see Materials and Methods), albeit not intermixed with unpracticed words, which would have allowed a stronger comparison (see Discussion).

Importantly, the ERPs and power spectra were similar when the practiced and unpracticed words were played (Fig. 3), but a clear LRP was observed for the wake list only $\left([2.750,3.200] \mathrm{s}, p_{\text {cluster }}=\right.$ 0.036 and $[3.230,3.900] \mathrm{s}, p_{\text {cluster }}=0.014, N=14$ participants $)$. In addition, a significant difference could be observed between the wake and REM lists $\left([3.230,4.120] \mathrm{s}, p_{\text {cluster }}=0.015\right)$. The presence 
a

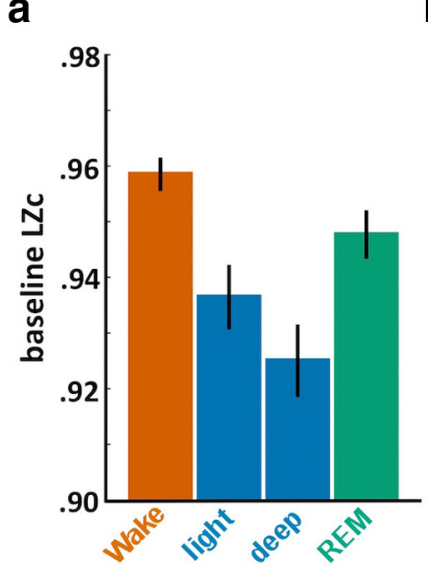

\section{b}

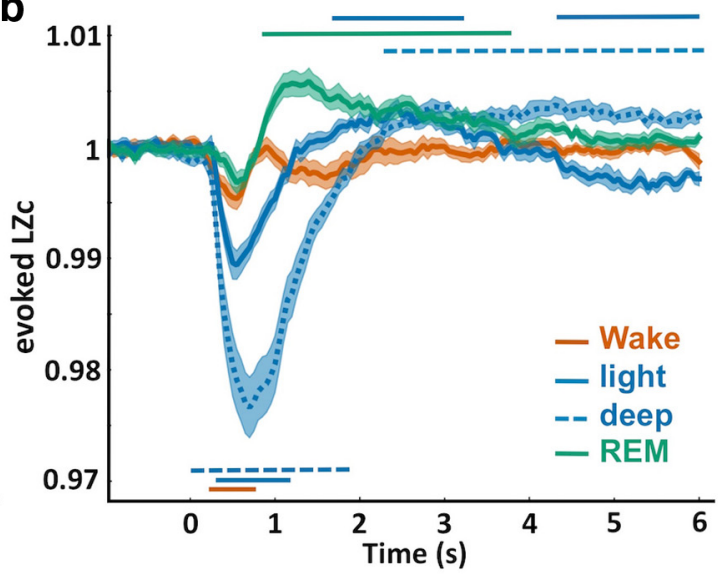

C
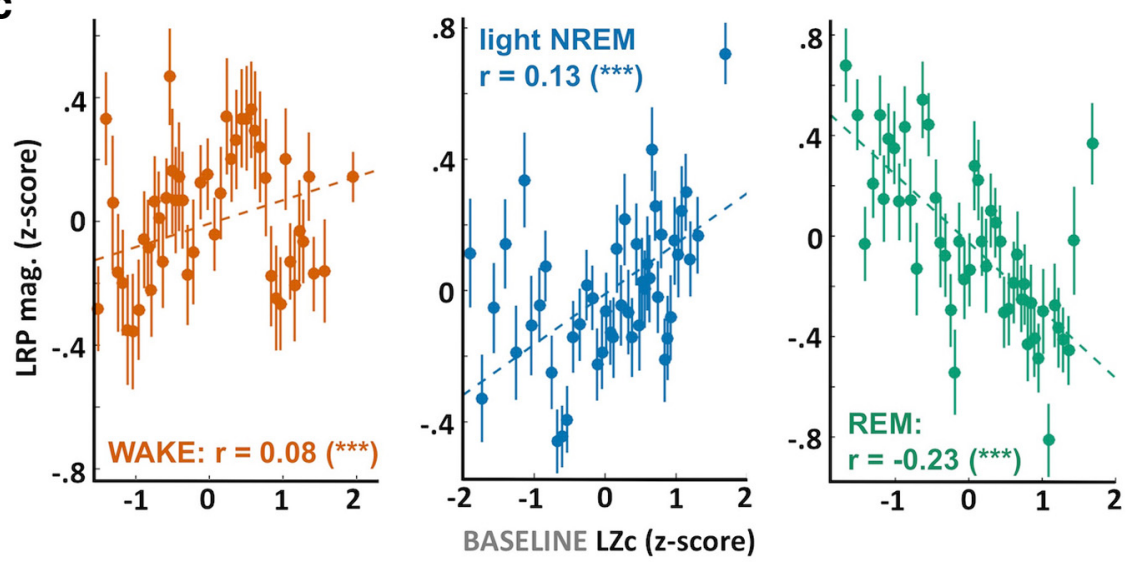

Figure 4. Lempel-Ziv complexity (LZC) across sleep stages and in relation to motor preparation indexes. $\boldsymbol{a}$, LZc extracted over the prestimulus activity $([-1.5,0] \mathrm{s})$ was averaged across trials scored as wakefulness, light NREM, deep NREM, and REM sleep. Error bars indicate the SEM computed across participants. LZc allowed to unambiguously separate the different vigilance states (one-way ANOVA: $F_{(3)}=9.67, p=2 \times 10^{-5}, N=18$ participants). Post hoc comparisons show highly significant differences with a gradual decrease in complexity: wake $>$ REM $>$ light NREM $>$ deep NREM (all paired $t$ tests, $p<0.005$ ). $\boldsymbol{b}$, LZc time course locked on stimulus onsets and expressed as a ratio of the baseline level $([-1.5,0] \mathrm{s})$. Stimuli robustly modulated the complexity of the EEG signal with an initial decrease after stimulus onset ( $p_{\text {cluster }}<0.05$, except for REM sleep). The initial decrease was followed by an increase in complexity in light NREM, deep NREM, and REM sleep $\left(p_{\text {cluster }}<0.05\right)$. c c Correlation between the baseline LZc (see $\boldsymbol{a}$ ) and the LRP magnitude computed across the entire night for wake (left), light NREM (middle), and REM sleep (right) trials. Correlation between the pairs of variables was assessed using the Pearson's method, which coefficients are displayed on each subplot along their significance levels. ${ }^{* *} p<0.005$. Dotted lines indicate the linear fit between the pairs of variables. Values were $z$-scored across trials for each participant before being aggregated across participants. Values were binned for visual purpose $(N=$ 50 bins on the sorted LZc values). Error bars indicate the SEM of the LRP magnitude for the corresponding bin.

modulation of $\mathrm{LZc}$ according to the vigilance state $\left(\right.$ ANOVA: $\mathrm{F}_{(3)}=9.67, p=2 \times$ $\left.10^{-5}\right)$. Such modulation indicates that, in wakefulness, the EEG signal is maximally complex (i.e., unpredictable), whereas, in deep NREM sleep, the EEG signal becomes the most predictable and therefore less complex according to the Lempel-Ziv algorithm. We could separate sleep and wake stages along a gradient matching the phenomenology associated with these states (Nir et al., 2013), confirming initial results in humans (Casali et al., 2013) and animals (Abásolo et al., 2015). Precisely, the baseline LZc was maximal in wakefulness (paired $t$ tests across participants with other sleep stages: all $p<0.005$ ) and minimal in deep NREM sleep. Light NREM and REM sleep had intermediary values, with REM sleep being the closest to wakefulness (REM vs wake: $t_{(17)}=-3.3$, $p=0.004$; REM vs light NREM: $t_{(17)}=$ 5.4, $p=5 \times 10^{-5}$; light vs deep NREM: $\left.t_{(17)}=9, p=7 \times 10^{-8}\right)$.

We then investigated whether this baseline LZc was predictive of LRP magnitude. LRP magnitude was extracted on the temporal windows in which a LRP was observed in wakefulness (Fig. 2a), light NREM sleep (Fig. 2c), and REM sleep (when practiced words were played; Fig. 3). Importantly, the baseline LZc was positively correlated with LRP magnitude in wakefulness and light NREM sleep (Pearsons's coefficient: $r=0.08$ and $0.13, p=$ 0.001 and $1 \times 10^{-13}$, across $N=2035$ and $N=3111$ samples in 17 and 15 participants, respectively), but this correlation was reversed in REM sleep $(r=-0.23$, $p=5 \times 10^{-24}, N=1937$ samples in 18 participants). Comparing $\mathrm{LZc}$ in REM sleep when practiced words were presented (LRP present) and when unpracticed words were presented (LRP absent) revealed again that the presence of the LRP was associated with a lower complex-

of an LRP for practiced words shows that sleepers can still map familiar stimuli with the correct response side in REM sleep, suggesting that part of the processing chain was conserved for practiced words. When repeating this procedure in NREM sleep, an LRP was also observed in light NREM for the words practiced in wake ([2.23, $2.9] \mathrm{s}, p_{\text {cluster }}=0.011, N=15$ participants) but not in deep NREM sleep despite the negative deflection visible in Figure 3.

\section{The processing of information depends on the degree of neural complexity}

We then set out to examine whether the overall complexity of the prestimulus EEG signal was predictive of sleepers' ability to process information up to the decision level. To do so, we used a recently developed approach (Casali et al., 2013) consisting in measuring the temporal predictability of the EEG signal by reducing it to a single value: the LZc (Ziv and Lempel, 1977). Applying this methodology to our data (Fig. 4a) revealed a strong ity (paired $t$ test: $t_{(17)}=3.5, p=0.004$ ). This result contrasts with the correlation observed in NREM sleep and wakefulness, suggesting an inverse relationship between levels of consciousness and the degree of connectedness to the environment (Sanders et al., 2012) in NREM and REM sleep.

We also examined how the $\mathrm{LZc}$ was modulated in response to stimuli above and beyond its baseline level (Fig. 4b). Stimulus onset was followed by a decrease in LZc (i.e., increase in signal predictability), potentially due to the stereotypical ERPs. And indeed, this decrease in LZc was more pronounced for the sleep stages in which high-amplitude ERPs were observed, such as in NREM sleep (Fig. 2). Accordingly, this decrease in LZc did not reach significance in REM sleep, a state in which auditory stimulation has less impact on brain activity (Bastuji and GarcíaLarrea, 1999; Picton, 2010). Interestingly, in NREM and REM sleep, this initial decrease was followed by an increase in $\mathrm{LZc}$ 
a

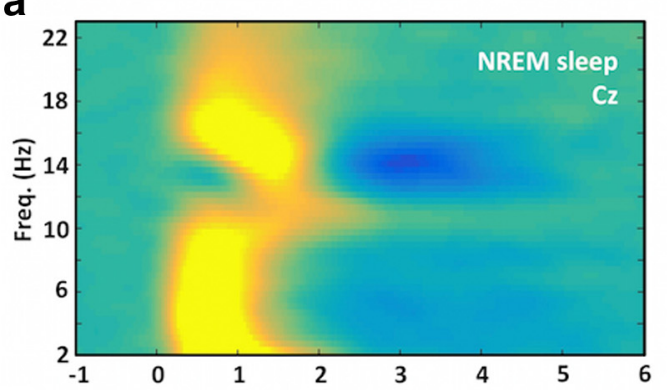

b

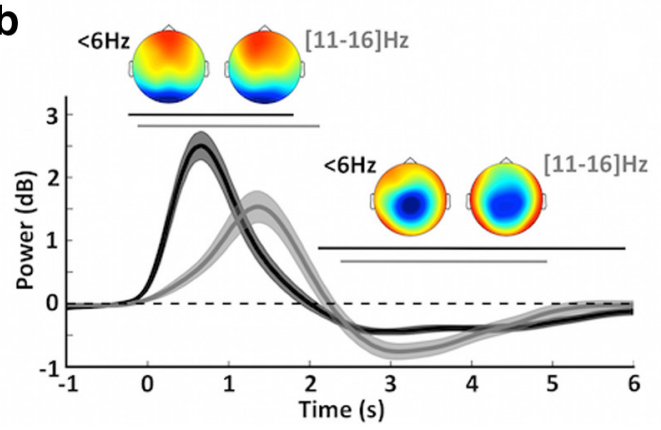

C

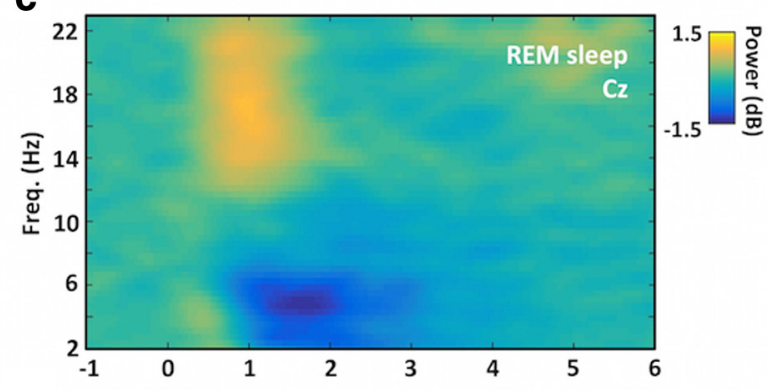

d

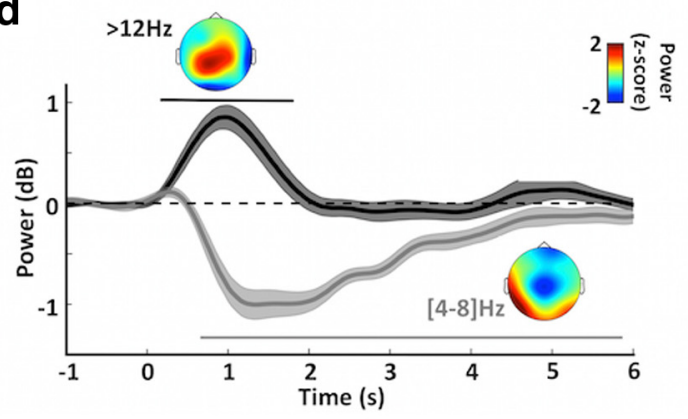

Figure 5. Local modulations of sleep rhythms in association to stimuli. $\boldsymbol{a}$, Time-frequency decomposition of the EEG signal recorded at Czin response to stimuli. The time-frequency decomposition was extracted for each trial in NREM sleep (light and deep) and averaged across participants $(N=18)$ (see Materials and Methods). Right after stimulus onset, a large increase in the low-frequency range $(<6 \mathrm{~Hz})$ and spindle range $([11,16] \mathrm{Hz})$ can be observed, which correspond to slow waves and spindles evoked by stimuli. Interestingly, these sleep rhythms were suppressed later on, at the time during which a LRP was observed in light NREM sleep (Fig. 2). This decrease was confirmed in $\boldsymbol{b}$ by examining the modulation of the power (at Cz) in these 2 frequency bands $(<6 \mathrm{~Hz}$ : slow-wave range, black curve; $[11,16] \mathrm{Hz}$ : spindle range, gray curve). Horizontal bars represent the significant clusters determined across participants $\left(p_{\text {cluster }}<0.05\right)$. Shaded areas represent the SEM computed across participants. Insets, Scalp topographies of the power within the slow-wave and spindle ranges at trial onset $([0,2] \mathrm{s})$ and during the LRP window ([2.9, 3.8] $\mathrm{s})$. Power was $z$-scored across sensors to emphasize regional differences. The decrease associated with the LRP is centrally distributed for slow waves and sleep spindles despite their originally frontal distribution, suggesting a local suppression of sleep rhythms. $\boldsymbol{c}, \boldsymbol{d}$, Same as $\boldsymbol{a}, \boldsymbol{b}$, except for REM sleep. Note the initial broadband increase in the higher frequency range $(>12 \mathrm{~Hz})$ and the decrease within the theta range $([4,8] \mathrm{Hz})$. Scalp topographies were computed by averaging power over the significant clusters $\left(p_{\text {cluster }}<0.05\right)$.

$\left(p_{\text {cluster }}<0.05\right)$, which could be interpreted as a stimulusinduced modulation of sleep depth.

\section{Sensory processes locally disrupt sleep rhythms}

So, are the LRPs observed in sleep and the associated increase in LZc reflecting the consequence of a partial awakening? To answer these questions, we examined the consequences of auditory stimulation on sleep itself. Figure $5 a$ shows the time-frequency decomposition of the EEG signal in response to sounds in NREM sleep (both light and deep NREM) for electrode Cz. Stimulus onset was accompanied by an increase within the slow-wave $\left(<6 \mathrm{~Hz}\right.$ : $p_{\text {cluster }}=0.001, N=18$ participants) and spindle $\left([11,16] \mathrm{Hz}: p_{\text {cluster }}=0.002\right)$ ranges (Fig. $5 b)$. This increase was followed by a decrease within these two ranges (<6 Hz: $[2.1,6.3] \mathrm{s} ;[11,16] \mathrm{Hz}:[2.4,5.0] \mathrm{s}$, both $\left.p_{\text {cluster }}<0.001\right)$, interestingly, at a time corresponding to the appearance of an LRP in light NREM sleep. This result suggests that the preparation of taskrelated responses translates into a local-in-time modulation of sleep rhythms and hence of sleep depth. Such modulation was also localin-space. Indeed, while the increase within the slow-wave and spindle range after stimulus onset showed a typical frontal topography (Fig. 5b), the following decrease had a different topography and was prominent over central electrodes (i.e., over the sensors showing the LRP). It is important to note that this decrease within the slow-wave and spindle ranges was not accompanied by an increase in frequency bands associated with wakefulness (alpha: $[9,11] \mathrm{Hz}$, beta: $>16 \mathrm{~Hz}$ ), which is concordant with the fact that trials with signs of arousal were discarded from our analyses. Thus, in NREM sleep, the processing of auditory information resulted in a local (in time and space) modulation of NREM oscillations in the absence of any observable trace of awakening on the scalp.
In REM sleep, brain responses were qualitatively different (Fig. $5 c, d$ ): auditory inputs clearly disturbed REM sleep with an initial increase in higher frequencies $\left(>12 \mathrm{~Hz}, p_{\text {cluster }}=0.01\right)$. This increase was followed by a sustained decrease within the theta range $\left([4,8] \mathrm{Hz}, p_{\text {cluster }}<0.001\right)$, reflecting a stimulusinduced perturbation of theta oscillations, which are a hallmark of REM sleep in animals (Buzsáki, 2006). Interestingly, these increases were both maximal over central electrodes where auditory and motor components are observed in wakefulness (Picton, 2010; Smulders et al., 2012). However, these transient markers of arousal did not lead to the appearance of a clear LRP (Fig. 2) unless practiced words were presented (Fig. 3).

\section{Neuronal bistability gates sensory processing in NREM sleep}

In NREM sleep, brain responses to stimuli were characterized by strong evoked potentials, such as the P200 and the N550 (Fig. 6a). These two potentials are of high interest because the P200 is thought to reflect an activation within the primary sensory cortices corresponding to the eliciting event (i.e., dependent of the sensory modality), whereas the N550 is thought to reflect a broad modality-independent neuronal silencing, maximal in frontal areas (Laurino et al., 2014; Halász, 2015). The respective scalp topographies of these potentials corroborated such view (Fig. $6 a$, right). According to this interpretation, the P200 could represent a marker of the brain's responsiveness to external events, whereas the N550 could be associated with sleep protection against external perturbations.

We thus examined whether the auditory responses (evokedpotentials associated with stimulus onset) were predictive of the appearance of an LRP. Interestingly, in light NREM sleep, the 
a

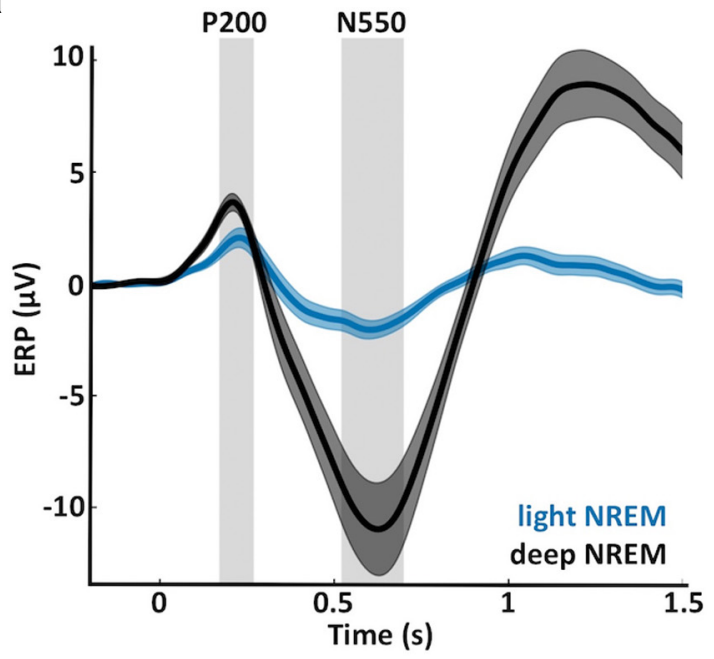

b

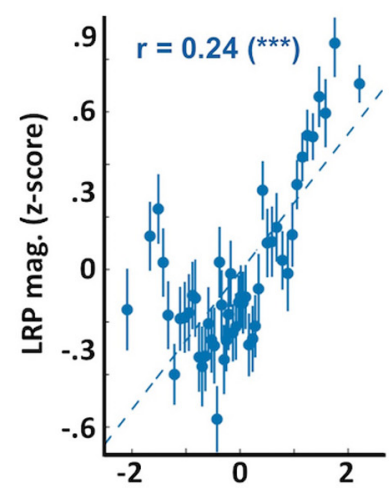

\section{light NREM}
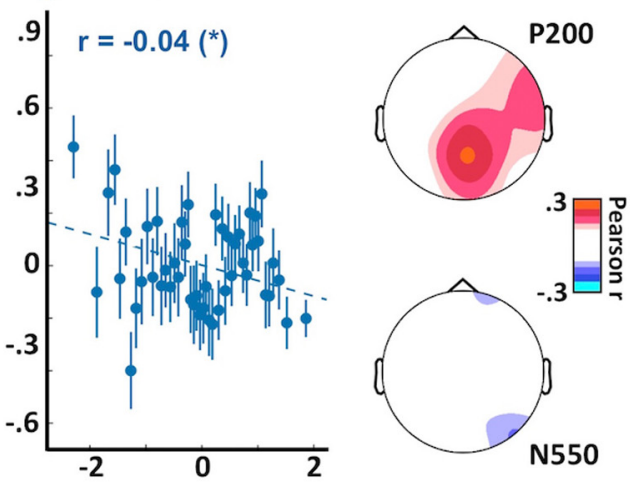

C

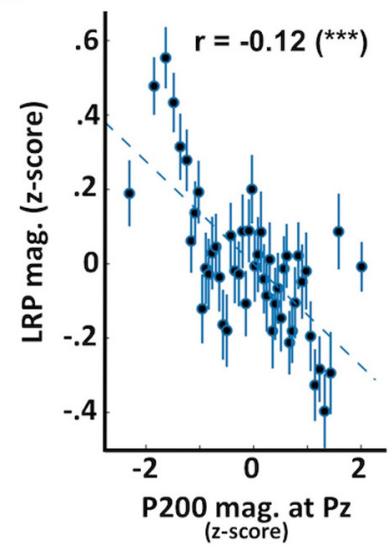

deep NREM
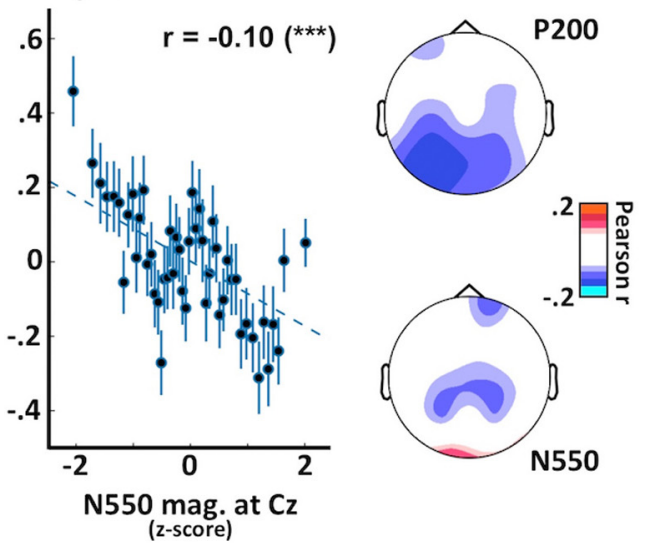

Figure 6. Neural bistability gates sensory processing in NREM sleep. $\boldsymbol{a}$, ERPs computed at C $z$ for trials in light NREM (blue curve) and deep NREM sleep (black). Two distinct potentials are clearly visible: a positivity $\sim 200 \mathrm{~ms}$ (P200) maximal at centroparietal electrodes (see scalp topography on the top right); and a negativity 550 ms (N550) predominant in deep NREM sleep (trials associated with slow waves) and maximal at frontal electrodes (see scalp topography on the bottom right). Shaded areas represent the SEM computed across participants $(N=18)$. Scalp topographies were established by averaging the voltage over windows around the two potentials of interest (see gray areas on ERP plot). These values were averaged across participants and $z$-scored across channels to emphasize regional differences. $\boldsymbol{b}$, Correlations between the LRP magnitude and the P200 magnitude (left) or the N550 magnitude (right, opposite of amplitude) for trials in light NREM sleep. The P200 and N550 magnitudes were computed at Pz and Cz, respectively. Correlation between the pairs of variables was assessed using Pearson's method, with coefficients displayed on each subplot along with their significance levels. ${ }^{* *} p<0.005$. ${ }^{*} p<$ 0.05 . Dotted lines indicate the linear fit between the two pairs of variables. Values were $z$-scored across trials for each participant before being aggregated across participants. Values were binned for visual purpose ( $N=50$ bins on the sorted $x$-axis variable). Error bars indicate the SEM of the LRP magnitude for the corresponding bin. Scalp topographies on the right represent the Pearson coefficients computed for each sensor (nonsignificant coefficients were set to $0, p>0.05$, FDR corrected for multiple comparisons). c, Same as in $\boldsymbol{b}$ for trials in deep NREM sleep. The reversal of the relationship between the LRP and the P200 from light to deep NREM paralleled with the appearance of a large N550, showing a suppressive effect on LRP magnitude.
N550 was largely reduced, which is in line with the fact that only trials without slow waves were included. The P200 was also reduced. When examining the relationship between these events and the LRP magnitude in light NREM sleep (Fig. 6b), a positive relationship was found between LRP magnitude and the P200 (Pearson coefficient at Pz: $r=0.24, p=6 \times 10^{-42}$, $N=3111$ samples in 15 participants). This correlation was maximal over centroparietal electrodes. Thus, the larger the P200, the larger the LRP in light NREM sleep. A much less robust correlation was observed with the magnitude of the N550 ( $r=-0.04, p=0.02)$, which could be explained by the fact that, in light NREM, the N550 was almost abolished.

On the other hand, in deep NREM sleep, there was a clear negative correlation between the N550 and the LRP magnitude (Fig. $6 c$, Pearson coefficient at Cz: $r=-0.10, p=10^{-4}, N=6037$ samples in 18 participants). Thus, the more pronounced the N550, the smaller the LRP in deep NREM sleep. Interestingly, the relationship between LRP magnitude and the P200 was reversed between light and deep NREM sleep. Contrary to light NREM sleep, the LRP magnitude was negatively correlated with LRP magnitude (Pearson coefficient at Cz: $r=-0.12, p=10^{-20}$ ) despite the fact that the P200 increases in amplitude from light to deep NREM sleep (Fig. 6a), which should favor sensory processing according to the positive relationship observed in light NREM sleep. Such reversal could be due to the fact that the P200 activation can trigger a down state (N550) in deeper stages of NREM sleep, which would ultimately inhibit information processing and reverse the relationship between the P200 and the LRP (see Discussion).

\section{Auditory evoked potentials are predictive of motor preparation in REM sleep}

We applied the same approach to REM sleep. Auditory stimuli in REM sleep also evoked archetypal potentials: a positivity $\sim 200 \mathrm{~ms}$ followed by a negativity $\sim 500$ ms (Fig. 7a). However, the topography and temporal profile of these potentials are quite different from NREM potentials. In REM sleep, the P200 is usually associated with the wake auditory P200, generated over the primary and secondary cortices (Picton, 2010). The scalp topography of the P200 with a maximum over central electrodes is concordant with this view. Contrary to light NREM sleep, the P200 was negatively correlated with the 
LRP magnitude in REM sleep (Pearson coefficient at Pz: $r=-0.14, p=4 \times$ $10^{-11}, N=2622$ samples in 18 participants; Fig. $7 b$ ). This negative correlation was maximal over occipital electrodes.

The N500, a potential that is prominent over occipital electrodes in REM sleep, was positively correlated with LRP magnitude (Pearson coefficient at Pz: $r=$ $0.15, p=3 \times 10^{-14}$; Fig. $7 c$ ), an opposite relationship compared with the one found for the N550 potential in NREM sleep. Similar correlations were observed when considering the REM trials in which words practiced in wake were played (P200: $r=-0.12, p=8 \times 10^{-4}$; N500: $\left.r=0.15, p=7 \times 10^{-15}\right)$. These results indicate that the evoked potentials associated with auditory stimuli are predictive of later and more complex stages of processing (here motor preparation) and suggest that these auditory potentials are not an unspecific reaction to external stimuli but rather reflect cortex's responsiveness to these inputs.

\section{Markers of responsiveness are modulated within sleep cycles}

The presence of LRP in light NREM but not deep NREM sleep or REM sleep (when unpracticed words are presented) could be due to the fact that light NREM sleep is more pervasive to external information. Another interpretation would be that the ability to prepare for the adequate motor response slowly decays with the time spent asleep. With light NREM sleep occurring first, the LRP would be prominent in this state. To test this possibility, we examined how the LRP magnitude was modulated within sleep cycles. Sleep cycles were detected using participant's hypnograms (see Materials and Methods). For each cycle, we retrieved the dynamics of the LZc, the slow-wave power ( $\partial$ power, a proxy for slow-wave density), and the LRP magnitude in the NREM part of the cycle (Fig. 8). In accordance with the archetypal profile of sleep cycles, slowwave power density gradually increased at the beginning of the sleep cycle (descending slope) but decreased toward the end of the cycle (ascending slope and transition to REM sleep) (Halász, 2015). The LZc mirrored this pattern with a gradual decrease in overall complexity followed by a steep increase. Interestingly, the LRP magnitude followed the LZc dynamics and increased again toward the end of the cycle ( $U$ shape). This result suggests that the capacity to process information is dynamically related to signal complexity and neural bistability. Thus, the beginning and end of NREM episodes might represent temporal windows in which monitoring of the surrounding environment is possible.

We also examined the effect of the progression within the night on LRP magnitude. To do so, each night was divided into
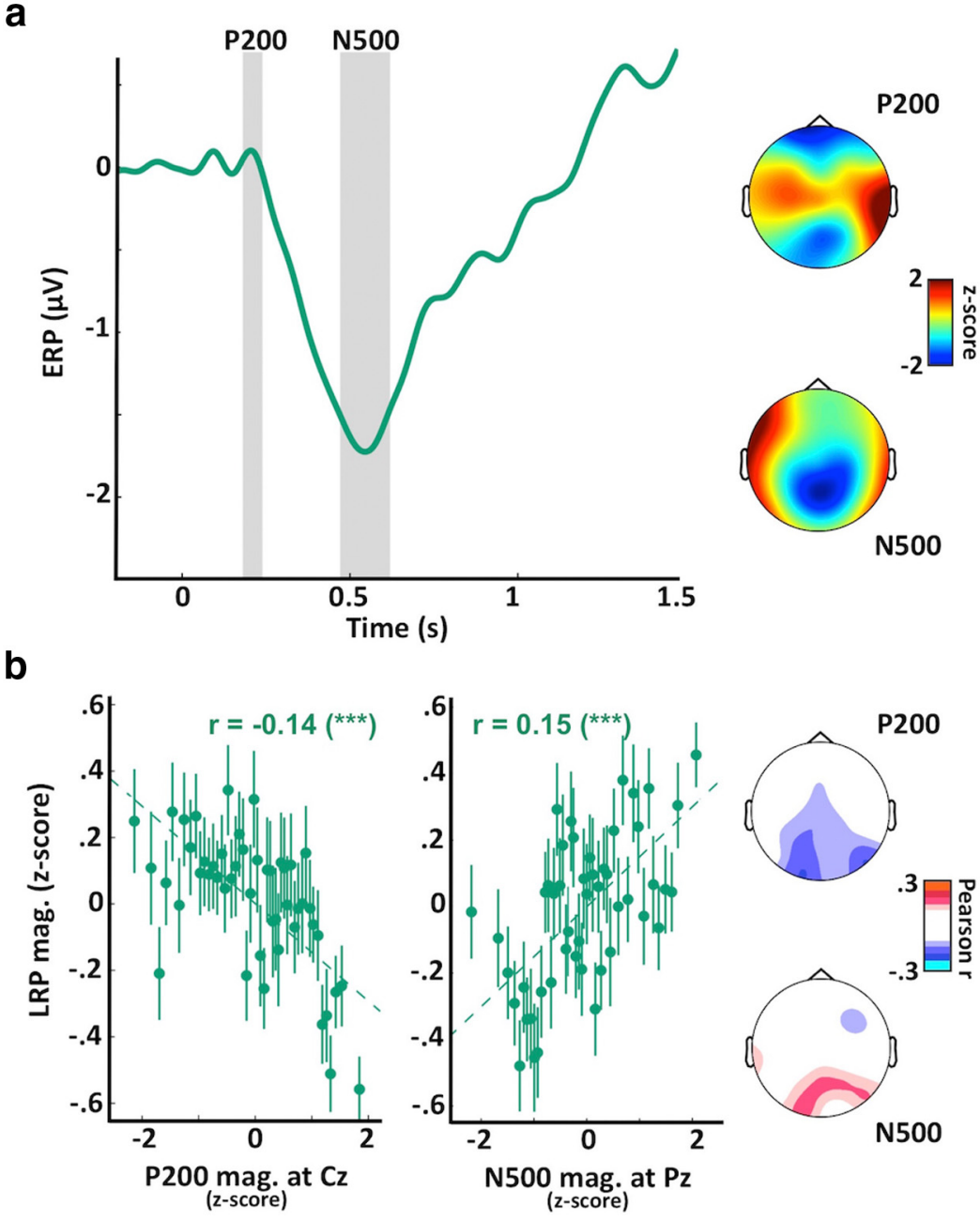

Figure 7. Evoked responses to sounds correlate with LRP magnitude in REM sleep. $\boldsymbol{a}$, ERPs computed at Cz for trials in REM sleep. Two distinct potentials are again visible: a positivity $\sim 200 \mathrm{~ms}$ (P200) maximal at central electrodes (see scalp topography on the right); and a negativity $\sim 500 \mathrm{~ms}$ (N500) maximal at parietal electrodes (see scalp topography on the bottom right). Scalp were established by averaging the voltage over windows around the two potentials of interest (see gray areas on ERP plot). These values were averaged across participants and z-scored across channels to emphasize regional differences. These two cance levels. ${ }^{* *} p<0.005$. Dotted lines indicate the linear fit between the two pairs of variables. Values were z-scored across trials for each participant before being aggregated across participants. Values were binned for visual purpose $(N=50$ bins on the sorted represent the Pearson coefficients computed for each sensor (nonsignificant coefficients were set to $0, p>0.05$, FDR corrected for multiple comparisons). Similar correlations were obtained when focusing on practiced words (data not shown).

thirds and the LRP magnitude was computed in light NREM, deep NREM, and REM trials in each third separately. In light NREM sleep, a linear decrease was observed across the night (linear regression: $\beta=-0.41, p=0.002, N=15$ participants). A post hoc comparison showed a highly significant difference between the LRP magnitude in the first and last third of the night $\left(t_{(14)}=4.03, p=0.001\right)$ with only the first third of the night showing a significant $\operatorname{LRP}\left(t_{(14)}=3.15, p=\right.$ $0.007)$. Such within-nights effect was not observed in deep NREM or REM trials (linear regressions: $\beta=-0.20, p=0.44$ and $\beta=-0.08, p=0.58$, respectively). However, because sleep lists were repeated across the night, it is impossible to disentangle in this study a potential effect of early and late sleep from an effect of word-repetition. 


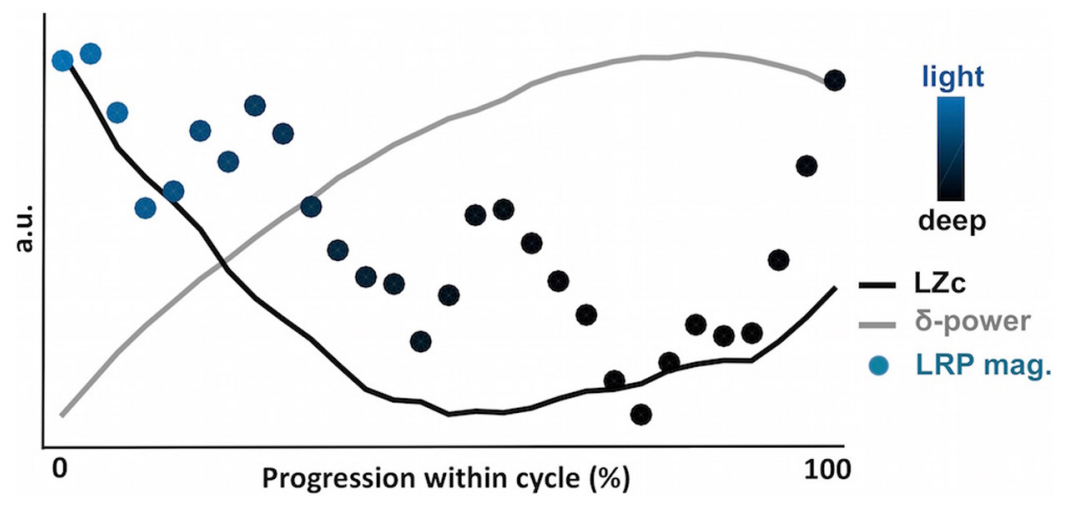

Figure 8. The ability to process information is dynamically modulated within sleep cycles. Modulation of the LRP magnitude (colored dots), the LZc (black curve), and the $\partial$-power (gray curve) within the NREM sleep part of sleep cycles. Colors of dots (LRP magnitude) represent the proportion of light and deep NREM trials included in the corresponding bin. A classical increase in $\partial$-power (a proxy for slow-wave density) is observed corresponding to the transition from light to deep NREM sleep. This increase in $\partial$-power is accompanied by a decrease in LZc and LRP magnitude. Both LZc and LRP have the tendency to increase again at the end of the NREM cycle, paralleling the transition from deep NREM sleep to REM sleep. LRP, LZc, and $\delta$ values were estimated within each sleep cycle on fixed windows (see Materials and Methods). Sleep cycles were then binned $(N=30)$ so as to average cycles with different durations, and values were normalized across the entire cycle to better visualize the dynamics of each variable of interest (expressed here in arbitrary units [a.u.]).

\section{Discussion}

\section{Complex and distributed processes in the sleeping brain}

Participants had to categorize spoken words based on their semantic category through lateralized hand responses while falling asleep. To assess participants' responsiveness, we computed an LRP (Smulders et al., 2012), which indicates whether brain activity is lateralized according to the expected response side. Accordingly, a negative deflection was observed in wakefulness around response time (Fig. 2a). This negativity was conserved in light NREM sleep when unpracticed words were played despite the absence of overt responses (Fig. 2c), replicating previous findings in naps (Kouider et al., 2014). Because the stimulus-response mapping was counterbalanced across participants, the presence of an LRP for novel words in light NREM sleep reflects the maintenance of complex and distributed processes going from the encoding of the auditory information to the preparation of the appropriate motor response. The maintenance of such complex processing chain could be explained by the automation of the task-set during wakefulness (Kouider et al., 2014).

No LRP was observed in deep NREM sleep or REM sleep (for unpracticed words), suggesting that part of this chain is disrupted in these stages. However, when considering practiced words (wake list, Fig. 1), an LRP was observed in REM sleep (Fig. 3). The presence of an LRP for practiced words only could be interpreted as a failure to access the meaning of novel words during the REM period. Under such circumstances, sleepers could still rely on stimulus-response contingencies learned during wakefulness for practiced words. However, because the practiced and unpracticed words were not intermixed, we cannot rule out the possibility that the presence or absence of an LRP stems from a difference in terms of sleep (i.e., sleep depth) rather than stimulus type (practiced or not).

\section{The complexity of brain dynamics interacts with the processing of the environment}

Certain periods of sleep proved more propitious for the induction of task-dependent responses. To understand the relationship between the background neural activity and brain's responsiveness, we computed the temporal predictability of the EEG signal using LZc (Ziv and Lempel, 1977). LZc has been used to determine the level of consciousness during anesthesia or in brain-damaged patients (Casali et al., 2013; Sarasso et al., 2015; Schartner et al., 2015). We confirmed previous findings in sleep (Casali et al., 2013; Abásolo et al., 2015) and LZc matched sleep's phenomenology (Nir et al., 2013), dreams (i.e., conscious contents) being more frequent and complex in REM compared with light NREM but quite rare in deep NREM sleep.

LZc also predicted the LRP magnitude (Fig. 4c). In light NREM sleep, an increased propensity to respond to external input was associated with higher levels of complexity before stimulus onset. In REM sleep, however, this relationship was reversed, which could be related to the peculiarity of REM sleep: a state of consciousness disconnected from the environment (Hobson, 2009). This result emphasizes the difference between consciousness and responsiveness as observed in anesthesia (Sanders et al., 2012).

\section{Neuronal bistability and the gating of sensory processing}

In contrast with light sleep, no LRP was observed in deep NREM sleep. Light and deep NREM sleep were defined based on the presence or absence of slow oscillations associated with stimuli. Accordingly, light NREM showed a drastically reduced N550 potential (Figs. 2, 6), a potential usually linked to the down states of stimuli-evoked K-complexes (Bastien et al., 2002). Down states represent episodes of neuronal silencing (Steriade, 2003) and perturb the encoding and integration of information (Schabus et al., 2012; Pigorini et al., 2015). The presence of a N550 could therefore prevent the further processing of information and abolish the LRP in deep NREM sleep. This hypothesis is buttressed by the positive correlation between the N550 and LRP magnitudes (Fig. 6c).

In light NREM sleep, stimulus-evoked potentials showed an initial P200 correlating positively with LRP magnitude (Fig. 6b). The P200 has been interpreted as a local modality-dependent excitation (Laurino et al., 2014) and could reflect brain's responsiveness to external inputs. However, in deep NREM sleep, the P200 was associated with LRP suppression (Fig. 6c). We interpret this reversal as the consequence of an increased neuronal bistability from light to deep NREM sleep.

Indeed, in deep NREM sleep, cortical neurons exhibit more down states (Steriade, 2003). Because a local excitation can trigger a down state (Sanchez-Vives et al., 2010; Menicucci et al., 2013), in deep NREM sleep, the P200 could ignite a down state in frontal areas that would shut down in turn any further processing. Thus, depending on sleep depth and neuronal bistability, the relationship of the P200 with sensory processing would reverse: from a potentiating activation to a suppressive mechanism. Brain responses to sounds could therefore follow a self-regulated process (Halász, 2015), whereby sensory activations can be retroactively suppressed. Thus, in NREM sleep, a reactive cortical gating (Tononi and Massimini, 2008), rather than a default thalamic one (McCormick and Bal, 1994), seems to take place. 


\section{Could dreams gate sensory processing in REM sleep?}

The mechanisms underlying sensory decoupling in REM sleep are still unknown. The recovery of a wake-like brain activity and consciousness should favor the processing of external information, and yet external inputs are rarely integrated in dream scenery (Nir and Tononi, 2010). A recent study described the presence of slow waves in REM sleep restricted to the superficial layers of primary sensory cortices (Funk et al., 2016). These slow waves could act as a gating mechanism because superficial cortical layers are the main targets of thalamic sensory relays (Jones, 2007). We showed here, however, that stimuli had a clear impact on cortical activity: signs of arousals could be observed after stimulus onset (Fig. $5 c, d$ ) as well as robust auditory potentials (Fig. 7). These activations indicate that sensory information did reach the cortex as previously suggested (Sallinen et al., 1996; Nir et al., 2015). However, no LRP was observed when novel words were played. A potential explanation is that stimulus-evoked perturbations were confined in sensory areas.

But what could limit distributed cortical processes if corticocortical connectivity is regained in REM compared to NREM sleep (Massimini et al., 2010)? We propose that sensory information may compete with endogenous contents (i.e., dreams). Indeed, wake-like endogenous activations have been observed in REM sleep (Louie and Wilson, 2001; Andrillon et al., 2015). These endogenous activations could compete for the brain's computational resources and interfere with the processing of external inputs. Accordingly, prestimulus complexity had a negative impact on LRP magnitude (Fig. 4). This "informational gating" could be mediated by a domination of top-down signaling at the expense of bottom-up processes (Nir and Tononi, 2010). Further investigations are needed to investigate the relationship between oneiric contents and sensory disconnection.

\section{Consequences of external stimulations on sleep}

How did stimulation affect sleep? In NREM sleep, we observed a two-step response (Fig. 5a,b). Right after stimulus onset, power increased within the slow-wave and spindle ranges in accordance with the fact that sleep rhythms can be evoked by external stimulations (Halász, 2015). These increases were maximal over frontal electrodes and could correspond to a protective mechanisms ensuring that sleep is preserved (Halász et al., 2014). Likewise, the suppressive N550 (Fig. 6) was maximal at frontal electrodes. After this initial increase, slow waves and spindle power were on the contrary suppressed. The associated spatial and temporal distributions overlap with the time and location of the LRP (Fig. 2c) and the increase in LZc following stimulus presentation (Fig. 4b).

A local dampening of sleep depth, accompanying sensory processing, thus followed the initial protective response. No sign of arousal was observed, but the absence of arousal at the scalp level does not preclude local awakening in motor or sensory areas (Nobili et al., 2011; Peter-Derex et al., 2015). The local modulation of sleep depth associated with sensory processing could therefore reflect, at the scalp level, the occurrence of local wake within sleep (Nobili et al., 2012). In REM sleep, auditory stimulations also perturbed theta oscillations (Fig. $5 c, d$ ), a hallmark of REM sleep (Buzsáki, 2006). Signs of cortical activation (increase in the high-frequency range) could also be observed after stimulus onset. Once again, these perturbations were maximal over central electrodes, potentially revealing the recruitment of auditory areas.

\section{Responsiveness is dynamically modulated during sleep cycles} We also explored the dynamics of neural complexity, motor preparation, and $\partial$-power (a proxy for slow-wave density) across sleep cycles. Both the LZc and $\partial$-power dynamics reflected sleep cycles' structure, with an initial descent toward deep NREM sleep (increase in $\partial$-power, decrease of LZc) followed by a steep dampening of NREM sleep at the transition with REM sleep. The LRP magnitude was also robustly modulated within sleep cycles and paralleled complexity's dynamics. Crucially, LRP magnitude increased toward the end of sleep cycles. Thus, the LRP does not slowly decay after cycle onset but is tightly linked to sleep depth. This fragility of sleep at the transition with REM sleep could be instrumental in allowing the brain to "decide" whether to stay asleep or to wake up (Halász et al., 2004). This modulation of responsiveness during NREM sleep cycles is reminiscent of a model (Halász and Bódizs, 2013) in which the descending slope of the NREM cycle is gradually dominated by sleep-promoting mechanisms (such as evoked slow waves, as suggested by Fig. 6), whereas the ascending slope is characterized by the emergence of wake-promoting mechanisms, which could explain the recovery of the responsiveness marker toward the end of the NREM episode (Fig. 8). Interestingly, such a model would predict that external information would be better processed at the end of the NREM sleep cycle compared with the beginning, sleep depth being kept equal. Further research would be needed to confirm such a hypothesis.

Overall, our data suggest that sleepers can complexly and flexibly process information during certain sleep stages. This ability is self-regulated by the neural dynamics, allowing the sleeping brain to manage the challenging tradeoff between sensory decoupling and monitoring its environment.

\section{References}

Abásolo D, Simons S, Morgado da Silva R, Tononi G, Vyazovskiy VV (2015) Lempel-Ziv complexity of cortical activity during sleep and waking in rats. J Neurophysiol 113:2742-2752. CrossRef Medline

Andrillon T, Nir Y, Cirelli C, Tononi G, Fried I (2015) Single-neuron activity and eye movements during human REM sleep and awake vision. Nat Commun 6:7884. CrossRef Medline

Arzi A, Shedlesky L, Ben-Shaul M, Nasser K, Oksenberg A, Hairston IS, Sobel N (2012) Humans can learn new information during sleep. Nat Neurosci 15:1460-1465. CrossRef Medline

Bastien CH, Ladouceur C, Campbell KB (2000) EEG characteristics prior to and following the evoked K-xomplex. Can J Exp Psychol 54:255-265. Medline

Bastien CH, Crowley KE, Colrain IM (2002) Evoked potential components unique to non-REM sleep: relationship to evoked K-complexes and vertex sharp waves. Int J Psychophysiol 46:257-274. CrossRef Medline

Bastuji H, García-Larrea L (1999) Evoked potentials as a tool for the investigation of human sleep. Sleep Med Rev 3:23-45. CrossRef Medline

Benjamini Y, Yekutieli D (2011) The control of the false discovery rate in multiple testing under dependency. Ann Stat 29:1165-1188.

Brainard DH (1997) The Psychophysics Toolbox. Spat Vis 10:433-436. CrossRef Medline

Buzsáki G (2006) Rhythms of the brain. Oxford: Oxford UP

Casali AG, Gosseries O, Rosanova M, Boly M, Sarasso S, Casali KR, Casarotto S, Bruno MA, Laureys S, Tononi G, Massimini M (2013) A theoretically based index of consciousness independent of sensory processing and behavior. Sci Transl Med 5:198ra105. CrossRef Medline

Cash SS, Halgren E, Dehghani N, Rossetti AO, Thesen T, Wang C, Devinsky O, Kuzniecky R, Doyle W, Madsen JR, Bromfield E, Eross L, Halász P, Karmos G, Csercsa R, Wittner L, Ulbert I (2009) The human K-complex represents an isolated cortical down state. Science 324:1084-1087. CrossRef Medline

de Lavilléon G, Lacroix MM, Rondi-Reig L, Benchenane K (2015) Explicit memory creation during sleep demonstrates a causal role of place cells in navigation. Nat Neurosci 18:493-495. CrossRef Medline

Delorme A, Makeig S (2004) EEGLAB: an open source toolbox for analysis 
of single-trial EEG dynamics including independent component analysis. J Neurosci Methods 134:9-21. CrossRef Medline

Destexhe A, Hughes SW, Rudolph M, Crunelli V (2007) Are corticothalamic "up" states fragments of wakefulness? Trends Neurosci 30:334-342. CrossRef Medline

Ermis U, Krakow K, Voss U (2010) Arousal thresholds during human tonic and phasic REM sleep. J Sleep Res 19:400-406. CrossRef Medline

Formby D (1967) Maternal recognition of infant's cry. Dev Med Child Neurol 9:293-298. CrossRef Medline

Funk CM, Honjoh S, Rodriguez AV, Cirelli C, Tononi G (2016) Local slow waves in superficial layers of primary cortical areas during REM sleep. Curr Biol 26:396-403. CrossRef Medline

Halász P (2005) K-complex, a reactive EEG graphoelement of NREM sleep: an old chap in a new garment. Sleep Med Rev 9:391-412. CrossRef Medline

Halász P (2015) The K-complex as a special reactive sleep slow wave: a theoretical update. Sleep Med Rev 29:34-40. CrossRef Medline

Halász P, Bódizs R (2013) Dynamic NREM sleep regulation models. In: Dynamic structure of NREM sleep, pp 7-11. London: Springer.

Halász P, Terzano M, Parrino L, Bódizs R (2004) The nature of arousal in sleep. J Sleep Res 13:1-23. CrossRef Medline

Halász P, Bódizs R, Parrino L, Terzano M (2014) Two features of sleep slow waves: homeostatic and reactive aspects-from long term to instant sleep homeostasis. Sleep Med 15:1184-1195. CrossRef Medline

Hobson JA (2009) REM sleep and dreaming: towards a theory of protoconsciousness. Nat Rev Neurosci 10:803-813. CrossRef Medline

Hobson JA, Pace-Schott EF (2002) The cognitive neuroscience of sleep: neuronal systems, consciousness and learning. Nat Rev Neurosci 3: 679-693. CrossRef Medline

Ibáñez A, López V, Cornejo C (2006) ERPs and contextual semantic discrimination: degrees of congruence in wakefulness and sleep. Brain Lang 98:264-275. CrossRef Medline

Iber C, Ancoli-Israel S, Chesson A, Quan S (2007) The AASM manual for the scoring of sleep and associated events: rules, terminology and technical specifications. Westchester, IL: American Academy of Sleep Medicine.

Jones EG (2007) The thalamus, Ed 2. Cambridge: Cambridge UP.

Kothe CA, Makeig S (2013) BCILAB: a platform for brain-computer interface development. J Neural Eng 10:056014. CrossRef Medline

Kouider S, Andrillon T, Barbosa LS, Goupil L, Bekinschtein TA (2014) Inducing task-relevant responses to speech in the sleeping brain. Curr Biol 24:2208-2214. CrossRef Medline

Laurino M, Menicucci D, Piarulli A, Mastorci F, Bedini R, Allegrini P, Gemignani A (2014) Disentangling different functional roles of evoked K-complex components: mapping the sleeping brain while quenching sensory processing. Neuroimage 86:433-445. CrossRef Medline

Leuthold H (2003) Programming of expected and unexpected movements: effects on the onset of the lateralized readiness potential. Acta Psychol (Amst) 114:83-100. CrossRef Medline

Louie K, Wilson MA (2001) Temporally structured replay of awake hippocampal ensemble activity during rapid eye movement sleep. Neuron 29:145-156. CrossRef Medline

Maris E, Oostenveld R (2007) Nonparametric statistical testing of EEG- and MEG-data. J Neurosci Methods 164:177-190. CrossRef Medline

Masaki H, Wild-Wall N, Sangals J, Sommer W (2004) The functional locus of the lateralized readiness potential. Psychophysiology 41:220-230. CrossRef Medline

Massimini M, Ferrarelli F, Murphy M, Huber R, Riedner B, Casarotto S, Tononi G (2010) Cortical reactivity and effective connectivity during REM sleep in humans. Cogn Neurosci 1:176-183. CrossRef Medline

McCormick DA, Bal T (1994) Sensory gating mechanisms of the thalamus. Curr Opin Neurobiol 4:550-556. CrossRef Medline

Menicucci D, Piarulli A, Allegrini P, Laurino M, Mastorci F, Sebastiani L, Bedini R, Gemignani A (2013) Fragments of wake-like activity frame down states of sleep slow oscillations in humans: new vistas for studying homeostatic processes during sleep. Int J Psychophysiol 89:151-157. CrossRef Medline

New B, Pallier C, Brysbaert M, Ferrand L (2004) Lexique 2: a new French lexical database. Behav Res Methods Instrum Comput 36:516-524. CrossRef Medline

Nir Y, Tononi G (2010) Dreaming and the brain: from phenomenology to neurophysiology. Trends Cogn Sci 14:88-100. CrossRef Medline

Nir Y, Staba RJ, Andrillon T, Vyazovskiy VV, Cirelli C, Fried I, Tononi G
(2011) Regional slow waves and spindles in human sleep. Neuron 70: 153-169. CrossRef Medline

Nir Y, Massimini M, Boly M, Tononi G (2013a) Sleep and consciousness. In: Neuroimaging of consciousness (Cavanna AE, Nani A, Blumenfeld $\mathrm{H}$, Laureys S, eds), pp 133-182. Berlin: Springer.

Nir Y, Vyazovskiy VV, Cirelli C, Banks MI, Tononi G (2015) Auditory responses and stimulus-specific adaptation in rat auditory cortex are preserved across NREM and REM sleep. Cereb Cortex 25:1362-1378. CrossRef Medline

Nobili L, Ferrara M, Moroni F, De Gennaro L, Russo GL, Campus C, Cardinale F, De Carli F (2011) Dissociated wake-like and sleep-like electrocortical activity during sleep. Neuroimage 58:612-619. CrossRef Medline

Nobili L, De Gennaro L, Proserpio P, Moroni F, Sarasso S, Pigorini A, De Carli F, Ferrara M (2012) Local aspects of sleep. In: Progress in brain research, pp 219-232. Amsterdam: Elsevier.

Oostenveld R, Fries P, Maris E, Schoffelen JM (2011) FieldTrip: open source software for advanced analysis of MEG, EEG, and invasive electrophysiological data. Comput Intell Neurosci 2011:156869. CrossRef Medline

Oswald I, Taylor AM, Treisman M (1960) Discriminative responses to stimulation during human sleep. Brain 83:440-453. CrossRef Medline

Peigneux P, Laureys S, Delbeuck X, Maquet P (2001) Sleeping brain, learning brain: the role of sleep for memory systems. Neuroreport 12:A111A124. CrossRef Medline

Perrin F, García-Larrea L, Mauguière F, Bastuji H (1999) A differential brain response to the subject's own name persists during sleep. Clin Neurophysiol 110:2153-2164. CrossRef Medline

Peter-Derex L, Magnin M, Bastuji H (2015) Heterogeneity of arousals in human sleep: a stereo-electroencephalographic study. Neuroimage 123: 229-244. CrossRef Medline

Picton TW (2010) Human auditory evoked potentials. San Diego: Plural.

Pigorini A, Sarasso S, Proserpio P, Szymanski C, Arnulfo G, Casarotto S, Fecchio M, Rosanova M, Mariotti M, Lo Russo G, Palva JM, Nobili L, Massimini M (2015) Bistability breaks-off deterministic responses to intracortical stimulation during non-REM sleep. Neuroimage 112: 105-113. CrossRef Medline

Rasch B, Born J (2013) About sleep's role in memory. Physiol Rev 93: 681-766. CrossRef Medline

Riedner BA, Vyazovskiy VV, Huber R, Massimini M, Esser S, Murphy M, Tononi G (2007) Sleep homeostasis and cortical synchronization: III. A high-density EEG study of sleep slow waves in humans. Sleep 30:1643-1657. Medline

Ruby P, Caclin A, Boulet S, Delpuech C, Morlet D (2008) Odd sound processing in the sleeping brain. J Cogn Neurosci 20:296-311. CrossRef Medline

Sallinen M, Kaartinen J, Lyytinen H (1996) Processing of auditory stimuli during tonic and phasic periods of REM sleep as revealed by event-related brain potentials. J Sleep Res 5:220-228. CrossRef Medline

Sanchez-Vives MV, McCormick DA (2000) Cellular and network mechanisms of rhythmic recurrent activity in neocortex. Nat Neurosci 3:10271034. CrossRef Medline

Sanchez-Vives MV, Mattia M, Compte A, Perez-Zabalza M, Winograd M, Descalzo VF, Reig R (2010) Inhibitory modulation of cortical up states. J Neurophysiol 104:1314-1324. CrossRef Medline

Sanders RD, Tononi G, Laureys S, Sleigh JW (2012) Unresponsiveness $\neq$ unconsciousness. Anesthesiology 116:946-959. CrossRef Medline

Sarasso S, Boly M, Napolitani M, Gosseries O, Charland-Verville V, Casarotto S, Rosanova M, Casali AG, Brichant JF, Boveroux P, Rex S, Tononi G, Laureys S, Massimini M (2015) Consciousness and complexity during unresponsiveness induced by propofol, xenon, and ketamine. Curr Biol 25:3099-3105. CrossRef Medline

Schabus M, Dang-Vu TT, Heib DP, Boly M, Desseilles M, Vandewalle G, Schmidt C, Albouy G, Darsaud A, Gais S, Degueldre C, Balteau E, Phillips C, Luxen A, Maquet P (2012) The fate of incoming stimuli during NREM sleep is determined by spindles and the phase of the slow oscillation. Front Neurol 3:40. CrossRef Medline

Schartner M, Seth A, Noirhomme Q, Boly M, Bruno MA, Laureys S, Barrett A (2015) Complexity of multi-dimensional spontaneous EEG decreases during propofol induced general anaesthesia. PLoS One 10:e0133532. CrossRef Medline

Sela Y, Vyazovskiy VV, Cirelli C, Tononi G, Nir Y (2016) Responses in rat core auditory cortex are preserved during sleep spindle oscillations. Sleep 39:1069-1082. CrossRef Medline 
Siclari F, Bernardi G, Riedner BA, LaRocque JJ, Benca RM, Tononi G (2014) Two distinct synchronization processes in the transition to sleep: a highdensity electroencephalographic study. Sleep 37:1621-1637. CrossRef Medline

Smulders FT, Miller JO, Luck SJ, Kappenman E (2012) The lateralized readiness potential. In: Oxford handbook of event-related potential components, pp 209-229. Oxford, UK: Oxford Library of Psychology.

Steriade M (2003) Neuronal substrates of sleep and epilepsy, Ed 1. Cambridge: Cambridge UP.

Strauss M, Sitt JD, King JR, Elbaz M, Azizi L, Buiatti M, Naccache L, van Wassenhove V, Dehaene S (2015) Disruption of hierarchical predictive coding during sleep. Proc Natl Acad Sci U S A 112:E1353-E1362. CrossRef Medline
Töllner T, Rangelov D, Müller HJ (2012) How the speed of motor-response decisions, but not focal-attentional selection, differs as a function of task set and target prevalence. Proc Natl Acad Sci U S A 109:E1990-E1999. CrossRef Medline

Tononi G, Massimini M (2008) Why does consciousness fade in early sleep? Ann N Y Acad Sci 1129:330-334. CrossRef Medline

Vyazovskiy VV, Harris KD (2013) Sleep and the single neuron: the role of global slow oscillations in individual cell rest. Nat Rev Neurosci 14: 443-451. CrossRef Medline

Wauquier A, Aloe L, Declerck A (1995) K-complexes: are they signs of arousal or sleep protective? J Sleep Res 4:138-143. CrossRef Medline

Ziv J, Lempel A (1977) A universal algorithm for sequential data compression. IEEE Trans Inf Theory 23:337-343. CrossRef 